\title{
Journal of Molecular and Genetic Medicine
}

\section{The Long Non-Coding RNA XIST: A New Cornerstone in Carcinogenesis}

\section{Samir A, Salama E and El-Tayebi HM*}

Faculty of Pharmacy and Biotechnology, Department of Pharmacology and Toxicology, Genetic Pharmacology Research Group, German University in Cairo, Cairo, Egypt

\begin{abstract}
When an RNA does not code for a protein it will be nominated a non-coding RNA nevertheless this does not reflect that such RNA doesn't possess info or a certain role. Though claimed earlier to be evolutionary collected waste or noise related transcription rising from the initial gathering of genes, current evidence proposes that roles like physiology, pathology and cell development are played by non-coding RNAs which were claimed to be the genome unknown material. LncRNAs play pivotal roles in disease prognosis and pathogenesis especially in cancer. Epigenetic modifications do manipulate the gene function and hence contribute in cancer development. Furthermore, IncRNA XIST and TSIX plays a central role in X chromosome inactivation and are involved in countless diseases mainly cancer. In this review we summarize different epigenetic mechanisms, the different classifications of ncRNAs, IncRNAs functions and involvement in carcinogenesis as well as the two IncRNAs XIST and TSIX function and involvement in different cancers.
\end{abstract}

Keywords: Epigenetics; ncRNAs; lncRNA; XIST and TSIX in cancers

\section{Introduction to Epigenetics}

Epigenetics is defined as the change in phenotype not in genotype which means the change in gene expression not in DNA sequence and in simplified term, it is the study of biological mechanisms that switch genes on and off [1]. Epigenetics is a natural and regular phenomenon but can also be affected by several factors including age, diseases and environmental factors. DNA is made up of approximately 3 billion bases (Adenine, Guanine, Cytosine and Thymine), within the 3 billion bases there are about 20,000 genes. So, cancer will be cured if we could map the reasons and impacts of the different combinations and if we could switch on or off the genes to keep the good and eliminate the bad [2].

Epigenetic modification can be terminated as differentiated cells or can result in disorders such as cancer and immune disorders [3]. Regarding immune disorders, there are several evidences showing that change of epigenetic control contributes to autoimmune disease. Recently, studies showed that DNA methylation contributed to the pathogenesis of lupus whose $\mathrm{T}$ cells exhibit decreased DNA methyltransferase activity and hypo-methylated DNA [4].

Dysregulation of this resulted in overexpression of methylationsensitive genes such as the leukocyte function-associated factor (LFA-1) which causes lupus-like autoimmunity [5]. As for the mental retardation disorders, epigenetic changes play an important role in several disorders such as ATR-X, Fragile-X, Prader-willi and Angelman syndromes. For example, Prader-willi and Angelman display an abnormal phenotype as a result of the deletion of paternal or maternal copy of gene, respectively [6]. The same gene on the corresponding chromosome cannot compensate for the deletion because it has been turned off by DNA methylation which is an epigenetic modification. Additionally, epigenetic errors play a role in the causation of complex adult neuropsychiatric disorders, several reports have associated schizophrenia and mood disorders with DNA arrangements that include DNMT genes [7]. DNMT1 is selectively overexpressed in gammaaminobutyric acid (GABA)-ergic interneurons of schizophrenic brains, whereas hyper-methylation has been shown to repress expression of Reelin (a protein required for normal neurotransmission and memory formation) in brain tissue from patients with schizophrenia, psychosis and bipolar disorders. Finally, cancer was the first human disease to be linked to epigenetics [8]. Epigenetics is a valuable science in the study of human development. Epigenetic marks control the expression of genes that function in embryonic development and other reprogramming events [9]. These include re-establishment of DNA methylation, genetic imprinting, X-chromosome inactivation, development of pluripotent stem cells and differentiation of somatic cells. The epigenomic state is dynamic, tightly regulated and any dysregulation of epigenetic patterns is observed in many human diseases and multiple types of cancers [4]. Epigenetic marks are associated with specific diseases; tools can be developed to diagnose patients and gauge the severity of disease. There is also a great interest in therapeutic epigenetics. Several drugs such as methyltransferase inhibitors and histone deacetylase inhibitors are already used in cancer treatment [4]. There are issues with specificity and efficacy of these drugs so further research into their mechanisms is needed to develop better therapeutic agents.

\section{Literature Review}

\section{Types of epigenetics}

Epigenetics describe regulation at a level above or in addition to those of genetic mechanisms. Among different types are DNA methylation, hydroxy-methylation, histone modification, chromatin remodeling and regulation of gene expression by a group of either small or long non-coding RNAs [10]. At least three modifications are currently needed to initiate and maintain epigenetic change to achieve gene silencing [11]. The difference between Epigenetic and genetic mechanisms is that epigenetic mechanisms do not involve a change to DNA sequence whereas genetic mechanisms involve primary DNA sequence and changes or mutations to these sequences. It involves the modification of DNA which results in changes to the conformation of DNA and accessibility of other factors to DNA.

*Corresponding author: Hend M. El-Tayebi, Faculty of Pharmacy and Biotechnology, Assistant Professor, Department of Pharmacology and Toxicology, Head of the Genetic Pharmacology Research Group, German University in Cairo, Cairo, Egypt, Tel: +202 27589990-8; E-mail: hend.saber@guc.edu.eg

Received May 31, 2018; Accepted June 15, 2018; Published June 19, 2018

Citation: Samir A, Salama E, El-Tayebi HM (2018) The Long Non-Coding RNA XIST: A New Cornerstone in Carcinogenesis. J Mol Genet Med 12: 356 doi:10.4172/1747-0862.1000356

Copyright: $\odot 2018$ Samir A, et al. This is an open-access article distributed under the terms of the Creative Commons Attribution License, which permits unrestricted use, distribution, and reproduction in any medium, provided the original author and source are credited 
DNA methylation: First, the most studied and well characterized epigenetic modification is DNA methylation. In this process, DNA is modified at the fifth carbon by covalent methylation of cytosine bases resulting in modifying the function of the genes and inhibit the transcription by preventing transcription factors and lead to changes in chromatin structure that restrict access of transcription factors to the gene promoter. This process is carried out by a well-known group of enzymes called DNA methyltransferases (DNMTs) [12]. In humans, methylation of genome takes place under the control of DNMT3a and DNMT3b during embryonic development, so they mediate the methylation process. However, the maintenance of the established patterns of DNA methylation is mediated by DNMT1 and DNMT $3 \mathrm{~b}$. Methylated DNA attracts methyl cytosine binding proteins resulting in chromatin condensation which leads to transcription and gene expression repression [13]. Equally important and coupled with DNA methylation is DNA demethylation, the removal of a methyl group. The demethylation process is necessary for epigenetic reprogramming of genes and is also directly involved in many important disease mechanisms such as tumor progression. Demethylation of DNA can either be active or passive, or combination of both [14]. Passive DNA demethylation usually takes place on newly synthesized DNA strands via DNMT1 during replication rounds. Active DNA demethylation mainly occurs by the removal of 5-methylcytosine through the sequential modification of cytosine bases that have been converted by ten-eleven translocation (TET) enzyme-mediated oxidation [11]. In mammals, only cytosines preceding guanines (CpG dinucleotides) are known to be highly methylated are underrepresented relative to other dinucleotide combinations and are widely dispersed throughout the human genome. The majority of CpGs is in non-coding regions and is typically methylated. However, many of the remaining $\mathrm{CpG}$ dinucleotides are found in clusters upstream of a gene's coding sequence. These CpG islands are typically unmethylated or hypomethylated to allow for the expression of downstream genes. DNA regions near CpG islands referred to as island "shores" are often methylated and may serve to adjust the expression levels of nearby genes [11].

DNA hypomethylation is common in cancer cells. This may cause carcinogenesis promotion by transcriptional activation of proto-oncogenes and induction of chromosome instability whereas hypermethylation of DNA results in tumor suppressor genes silencing [15]. So, it is worth mentioning that accumulation of genetic and epigenetic modifications may give rise for invasive or metastatic transformed cells from normal cells [16].

Gain of CpG methylation can also be a feature of cancer cells and as methylated cytosines are highly unstable bases this will predispose gene mutation as the methylated cytosines are often deaminated and converted to thymine which can lead to inactivation of tumor suppressor genes [17]. Additionally, DNA methylation may cause abnormal expression of "cancer-associated genes", subsequently epigenetic changes can be used as biomarkers for molecular diagnosis of early cancer. DNA methylation was first confirmed to occur in human cancer in 1983 and has since been observed in many other illnesses and health conditions [11].

Histone modification: Histone modification considered as a covalent post-translational modification which include methylation, acetylation, phosphorylation, ubiquitination and sumoylation [18]. The combinations of histone modifications either by chromatin structure alteration or recruitment of histone modifiers control gene expression. They may achieve different effects based on the type and location of the modification. Methylation and acetylation are the most common and characterized histone modifications. First, Histone acetylation takes place through addition of an acetyl group from acetyl coenzyme A. Histone acetylation is engaged in numerous regulatory functions of many cellular processes including chromatin transcription, gene silencing, cell cycle progression, apoptosis, differentiation, replication, and repair through the assistance of histone acetyltransferases (HATs) which control histone acetylation at lysine residues whereas histone deacetylation is controlled by histone deacetylases (HDACs) [19]. Acetylated histone residues can also act as pockets for other histone-modifying enzymes or chromatin remodeling factors that promote gene expression. Any disruption in the equilibrium process of histone acetylation has been associated with tumor formation. HDAC inhibitors are currently being developed to serve as anti-cancer agents [20]. Second, in histone methylation process, methyl groups from the S-adenosyl-L-methionine transferred to lysine or arginine residues of histone proteins by histone methyl transferases (HMTs). It may result in gene repression or activation according to the location of the methylated residue, the methylation of lysine involves both activation and repression of transcription, while arginine methylation is implicated only in activation of transcription [21].

Histone demethylation is carried out by histone demethylases. These demethylases have been found to have potential oncogenic functions and involvement in other pathological processes. The discovery of histone demethylases demonstrates that the histone methylation is not a permanent process, but it is dynamic. Inhibition of histone demethylases may lead to histone re-methylation at specific residues important for chromatin dynamics and gene expression. Furthermore, detection of the activity and inhibition of these enzymes would be important in elucidating mechanisms of epigenetic regulation of gene activation and silencing and may benefit cancer diagnostics and therapeutics [22]. Finally, histone phosphorylation is a post-translational modification that involves the addition of a phosphoryl group to histone which play an important role in chromatin remodeling. Histone phosphorylation participates in a well-known function during the chromatin condensation while cell division, DNA repair and transcriptional regulation are occurring $[18,23,24]$.

Chromatin modification: Another significant epigenetic process is chromatin modification. Chromatin is the complex of histone proteins and DNA that is tightly bundled to fit into the nucleus. The complex can be modified by substances such as acetyl groups, enzymes, and some forms of RNA such as microRNAs and small interfering RNAs. This modification alters chromatin structure to influence gene expression. Chromatin remodeling proteins affect chromatin structure in various ways. They can expose DNA wrapped in nucleosomes by sliding histones along the DNA or detach the histone completely from a DNA sequence. Additionally, they can substitute particular subunits of the histone octamer by histone variants. Tightly folded chromatin considered to be repressed whereas open chromatin considered being functional $[25,26]$.

\section{Non-coding RNAs}

RNAs, especially non-coding RNAs (ncRNAs) affect chromatin structure. Non-coding RNAs are transcribed from DNA then 5 capped, polyadenylated but remain untranslated. Among important ncRNAs that affect epigenetic regulatory process include microRNAs (miRNA), short interfering RNAs (siRNA), piwi interacting RNAs (piRNA) and long non-coding RNAs (lncRNA). Generally, ncRNAs undergo numerous regulatory functions at the transcriptional and post-transcriptional level. These ncRNAs can be classified into two main groups; the short ncRNAs (less than 30 nucleotides) and the long 
ncRNAs (more than 200 nucleotides). miRNAs, siRNAs and piRNAs considered as the major classes of short ncRNAs [27].

NcRNAs are demonstrating a pivotal role in heterochromatin formation, histone modification, DNA methylation and gene silencing [28]. First, miRNAs bind to a specific target with affinity in the $3^{\prime}$ UTR region on mRNA resulting in mRNA cleavage or translation repression by activating miRNA-induced silencing complex (miRISC) through which miRNAs act as post-transcriptional repressors of gene expression. This process is catalyzed by the endoribonuclease Dicer, which cleaves double stranded RNA (dsRNA), and is implicated in the emerging of the endogenous siRNAs [29]. Second, siRNAs interpose post-transcriptional gene silencing (PTGS) so they are believed to function as miRNAs. Moreover, heterochromatin formation have been also proved to be induced by siRNAs through binding together with RNA-induced transcriptional silencing (RITS) complex which leads to H3K9 methylation and chromatin condensation [30]. Finally, piRNAs are named so due to their interaction with the piwi family of proteins. The primary function of these RNA molecules involves chromatin regulation and suppression of transposon activity in germ and somatic cells. PiRNAs that are anti-sense to expressed transposons target and cleave the transposon in complexes with PIWI-proteins resulting in generation of piRNAs which target and cleave further transposons till the production of considerable amounts of piRNAs to increase transposon silencing and repression [31-34].

When an RNA doesn't code for a protein its usually nominated a non-coding RNA, nevertheless this does not denote that such RNAs do not own information or execute a certain role [31]. Earlier it was postulated that most of genetic info that defines the form and the phenotype is in form of proteins that possess varied catalytic, structural functions and controls the action of the individual in variable means. This assumption can be correct in eukaryotes and prokaryotes [35]. However, modern biology surprised us by the discovery that the human genome encodes only 20,000 protein-coding genes, representing less than $2 \%$ of the total genome sequence. Afterward, it was determined that at least $90 \%$ of the genome is actively transcribed. It was not just an assembly of genes that do encode for proteins and their splice alternatives as the human transcriptome was proved to be more composite in a way that the human transcriptome shows wide overlying, antisense and ncRNA expression [36,37].

Although claimed earlier to be spurious transcriptional noise or accumulated evolutionary debris arising from the early assembly of genes and/or the insertion of mobile genetic elements, current evidence suggests that the dark matter of the genome may play a major biological role in cellular development, physiology, and pathologies. Generally, the more complex an organism, the greater is its number of ncRNAs. Non-coding RNAs are grouped into two major classes based on transcript size; small ncRNAs and long ncRNAs.

Small non-coding RNAs: RNA intervention and posttranscriptional RNA expression suppression represents the two main roles of small non-coding RNAs. Small non-coding RNAs execute their roles through the help of Argonaute proteins family and through helping in the recognition of messenger RNAs or the genome of viruses or antigenomic RNAs which are nominated as complementary single stranded RNAs. Furthermore, Small non-coding RNAs helps in their translational suppression and their breakdown. Argonaute protein family are involved in pathways that will result into the silencing of the genes, through the guidance of small non-coding RNAs. SncRNAs being anchored into specific binding pockets, guides Argonaute proteins to target mRNA molecules for silencing or destruction [37]. Besides, controlling chromatin modifications was identified as a role of the small ncRNAs $[38,39]$. Small non-coding RNAs can be classified into two groups based on whether their biogenesis is Dicer (Double stranded RNA 3 ribonuclease) dependent or independent:

Dicer dependent: miRNAs, SiRNAs and in some cases Small nucleolar RNA (soRNAs).

Dicer independent: PIWI-interacting RNAs (piRNAs) (Table 1) [40].

\begin{tabular}{|c|c|c|}
\hline MicroRNAs & $\begin{array}{l}\text { MicroRNAs (miRNAs) are from } 18 \text { to } 25 \text { nucleotides, represents } 1 \text { to } 2 \% \text { from the human genome, regulates } 50 \% \text { of } \\
\text { genes that do encodes for proteins; guide the repression of translation, they do depend on Drosha and Dicer, they } \\
\text { cause initiation of various malignant diseases, Control many human development processes like differentiation, cell } \\
\text { death and growth. }\end{array}$ & {$[17,21-26]$} \\
\hline Small interfering RNAs & $\begin{array}{l}\text { Small interfering (siRNAs) 19-23 nt; are a result of Dicer action; guides the destruction of the targeted mRNA, involved } \\
\text { in the treatment of diseases and the silencing of genes post transcriptionally }\end{array}$ & {$[144-147]$} \\
\hline Piwi interacting RNAs & $\begin{array}{l}\text { Piwi interacting (piRNAs) } 26-30 \mathrm{nt} \text {; bind Piwi proteins; Dicer independent; RNAs present in groups within genome, } \\
\text { the relation between piRNAs and diseases has not been revealed yet, they are elaborated in processes like stem self- } \\
\text { renewal, retrotransposon silencing and germ cell development. }\end{array}$ & [149] \\
\hline Small nucleolar RNAs & $\begin{array}{l}\text { Small nucleolar (snoRNAs) } 60-300 \mathrm{nt} \text {; the nucleolus is their main location, bind snoRNP proteins, related to malignant } \\
\text { diseases development, responsible for other non-coding RNAs maturation. }\end{array}$ & {$[27,28,149-154]$} \\
\hline $\begin{array}{l}\text { Promoter associated small } \\
\text { RNAs }\end{array}$ & $\begin{array}{l}\text { Promoter associated (PASRs) 20-200 nt; possess modified ends which are } 5 \text { ' capped; coincide with small RNAs the } \\
\text { transcriptional start sites of protein- and non-coding genes, made from transcription of short capped transcripts. }\end{array}$ & {$[29-32,155]$} \\
\hline $\begin{array}{l}\text { Centromere repeat associated } \\
\text { small interacting RNAs }\end{array}$ & $\begin{array}{l}\text { Centromere repeat associated small interacting RNAs (crasiRNAs)34-42 nt; processed from long dsRNAs. the } \\
\text { relationship between crasiRNAs and diseases has not yet been discovered. }\end{array}$ & {$[156]$.} \\
\hline Telomeric specific small RNA & $\begin{array}{l}\text { Telomeric specific small RNA (tel-sRNAs) } 24 \mathrm{nt} \text {; Dicer independent; 2'-O-methylated at the 3' end. evolutionarily } \\
\text { conserved from protozoa to mammals; have not been described in human up to now relationship between tel-sRNAs } \\
\text { and diseases has not yet been discovered / epigenetic regulation. }\end{array}$ & {$[151]$.} \\
\hline Pyknons & $\begin{array}{l}\text { subset of patterns of variable length; form mosaics in untranslated and protein-coding regions; more frequently in } \\
\text { 3'UTR, related to cancer development and elaborated in communication of the cells, transcription control, signaling, } \\
\text { transport. }\end{array}$ & {$[157-159]$} \\
\hline
\end{tabular}

Table 1: Classes of small non-coding RNAs. 


\section{Family of sncRNAs}

miRNAs, siRNAs, piwi interacting RNAs (piRNAs), Small nucleolar RNA (snoRNAs), Promoter associated small RNAs (PASRs), Transcription initiation RNAs (tiRNAs), Centromere repeat associated small interacting RNAs (crasiRNAs), Telomere-specific small RNAs (tel-sRNAs) and pyknons.

MicroRNAs: MicroRNAs are evolutionary conserved single stranded RNA molecule formed of 18 to 25 nucleotides and involved in specific gene regulation [17]. MicroRNAs controls the activity of 50\% of protein coding genes and represents $1 \%$ to $2 \%$ of human genome [41-43].

The majority of miRNAs are located in intergenic regions however minority of MicroRNAs are found in intronic regions either in the sense or antisense orientation [43]. MiRNAs function resides in post transcriptional repression of targeted genes through a miRISC complex. The post transcriptional repression of target genes depends on base pair complementarity between mRNA of target gene and miRNA, as perfect complementarity allows Ago-catalysed cleavage of messenger RNA strand whereas the central mismatch results in cleavage exclusion of mRNA which is substituted by its translational repression. The translational control mechanism mediated by miRNA results in the reduction of the protein levels of target genes without affecting their mRNA [44,45]. MicroRNAs functions resides in cellular development processes like cell death, differentiation and growth in addition to their role in carcinogenesis development $[46,47]$.

Small interfering RNas: SiRNAs are two partly single stranded RNA which do have sequence complementarity, called the guide strand and the passenger strand and are considered as an additional group of posttranscriptional RNA silencing. They are formed from dsRNAs having an endogenous or exogenous source.

The dsRNAs are 19 to 23 nucleotides long and made by Dicer processing like miRNAs. One of the resulting single strands will enter into the RISC (RNA-induced silencing complex) in which it will direct the destruction of precise sequence of complementary target mRNAs rather than translational repression.

SiRNAs are used widely in trials involved in gene silencing and do represents recently a specific and important tool of targeted genes expression switch off as well as a hopeful instrument in molecular oncology. SiRNAs are utilized in cancer treatment through several strategies such as the suppression of upregulated cancer genes, delaying division of the cell through the interference with cyclins or encouraging cell death through hindering genes that interferes with apoptosis.

\section{Piwi proteins associated RNAs}

PiRNAs are long RNAs produced through a way that doesn't depend on dicer resulting in production of 24 to 32 nucleotides. PiRNAs have been elaborated in germ cell expansion, stem cell selfregeneration, retrotransposon silencing and in cancers development.

\section{Small nucleolar RNAs}

Small nucleolar RNAs length ranges from 60 to 300 nucleotides, positioned in the nucleolus, where the formation and organisation of ribosomal RNAs (rRNAs) which is located in the cytoplasm occurs [48], Small nucleolar RNAs are situated inside introns originating from genes that do encode for proteins and are the result of RNA polymerase 2 transcription nevertheless, they can be produced from introns belonging to longer ncRNA generators. Small nucleolar Rnas can be categorized into two major classes:
First class: Comprises the box D (CUGA) and C (RUGAUGA) motifs.

Second class: Characterized by presence of ACA elements and the box H (ANANNA).

In both groups of snoRNAs, the structural core motifs of the snoRNAs is formed through short stems that brings the conserved boxes close to one another, which coordinate the binding of specific proteins to form small nucleolar RNPs (snoRNPs) distinct for both groups [48,49].

SnoRNAs have diverse roles like the development of other non-coding RNAs, managing alteration of rRNA and snRNA post transcriptionally through 2-O-methylation and pseudo uridylation, miRNA-like function, cellular targeting of mRNA as well as their involvement in cancer development.

Promotor associated RNAs: Promotor associated RNAs are coming from eukaryotic promoters and their length varies from $18-200$ nucleotides, present in proximity to the start site of the transcription or the promoter however they are not linked with genes that do encode for proteins and they own the ability to control the transcription of genes encoding for proteins through directing complexes responsible for epigenetic silencing [50-52].

Human PASRs are lowly expressed, their number is related to their mRNA expression level and their promotor activity [53]. when they are deregulated this will result into cancer formation as they are linked to transcription regulation.

Centromere repeat associated small interacting RNAs: Centromere repeat associated small interacting RNAs (crasiRNAs) are small RNA ranging from 34 to 42 nucleotides, elaborated in the employment of heterochromatin or proteins related to centromere and with no functional activity in cancer activity to date.

Telomere specific small RNA: Tel-sRNAs are Dicer-independent, their 3 'end is $2-\mathrm{O}$ methylated, 24 nucleotides long RNAs. Tel-sRNAs are postulated to be subjected to epigenetic regulations as they are found overexpressed in cells that have null mutation of $\mathrm{H} 3 \mathrm{~K} 4$ methyltransferase MLL and under expressed in cells that have null mutations of histone H3K9 methyltransferase SUV39H. Tel-sRNAs execute a pivotal role in cancer development and underwrite to limitless replicative potential of tumour cells.

Pyknons: Pyknons are a subcategory of sequences with variable length, found in the 3'UTR of genes instead of further areas of the human genome. Pyknons are located in genes which are elaborated in exact processes like transcription, cell communication, signalling, regulation of transcription and transport. Pyknons includes $40 \%$ of the recognised miRNA orders, signifying conceivable connection with posttranscriptional gene quietening and RNA interfering as well as their associated with cancer biology.

Long non-coding RNAs: They are non-protein coding transcripts longer than 200 nucleotides that have been recently found as regulators in various biological processes and involvement in the pathogenesis of many diseases including cancers. They are poorly conserved between species and expressed in very low to moderate level. They are extremely varied in structure and function; therefore, sequence length and absence of protein coding capability are their only defining characteristics [53]

LncRNAs are processed in a similar way to mRNA. Moreover, nuclear or cytosolic fractions are sites where they can be found. They are often transcribed from protein coding loci, however, several lncRNA 
subclasses have been described with different origins (Table 2). Some of them which are composed of transposable genomic elements belong to introns or form a sense-antisense pair called a" natural antisense transcript (NAT) " [54].

One particular subgroup of lncRNAs, the large intergenic noncoding RNAs (lincRNAs), has been associated with chromatin modifying complexes which can target specific genome loci to promote epigenetic states. The majority of characterized lncRNAs are generated by the same transcriptional machinery as other mRNAs, as evidenced by RNA polymerase II occupancy and histone modifications associated with transcription initiation and elongation. These $\operatorname{lncRNAs}$ have a 5 terminal methyl guanosine cap and often spliced and polyadenylated [55]. Alternate pathways also contribute to the generation of lncRNAs which include a poorly characterized contingent of nonpolyadenylated lncRNAs likely expressed from RNA polymerase III promotors and lncRNAs that are excised during splicing and small nuclear RNA production. LncRNAs were firstly thought to be a transcription noise without a biological function. In the past decades, however accumulating evidences have indicated that lncRNAs involve a wide range of biological functions such as; X-chromosome inactivation, reprogramming stem cell pluripotency, precursor for siRNAs, regulation of immune response and carcinogenesis, direct transcriptional regulation, modulation of microRNA regulation and regulation of genomic imprinting [56]. LncRNAs have been suggested to modulate gene expression by guiding chromatin modifying complexes to specific sites in the genome, thereby influencing gene expression. One widely known example of this is the role of XIST in $\mathrm{X}$-chromosome inactivation (XCI), this process involves two lncRNAs; XIST and its anti- sense transcript TSIX, a negative regulator of XIST. Prior to differentiation, XIST and TSIX are actively transcribed due to $\mathrm{H} 3 \mathrm{~K} 4$ di-methylation of the XIST gene. In this state XCI is a random event. Upon differentiation, XIST expression is elevated resulting in XIST RNA coating the future inactive $\mathrm{X}$-chromosome which triggers extensive histone methylation and chromosome inactivation [57]. They are key players in the development of human cancers as abnormal expression of lncRNAs contributes to unrestricted growth and invasion of cancer cells and their dysregulation potentially play an oncogenic or tumor suppressive roles. LncRNAs have been also found to be differentially expressed in various types of cancers including leukemia, hepatocellular carcinoma and breast cancer [58].

They are biomarkers that can be used for current and future clinical diagnosis, therapeutics and prognosis. Continued investigation of lncRNAs will likely prove to be exceeding valuable as they may provide novel therapeutic targets for cancer [59-61].

\section{Unique features for lncRNA biogenesis}

a) Chromatin state: At the level of chromatin state, lncRNA genes have a higher enrichment of $\mathrm{H} 3 \mathrm{~K} 27 \mathrm{ac}$ and are more strongly repressed by certain chromatin remodeling complexes such as Swr1 and Rsc [56].

b) Transcription initiation and direction: IncRNA transcription is enriched for $\mathrm{H} 3 \mathrm{~K} 56 \mathrm{ac}$ and phosphorylation of RNA polymerase II. Transcription in the divergent direction is further enhanced by the SWI/SNF proteins and repressed by CAF-1.

c) Transcriptional elongation: They are more regulated strongly by DICER1 and MYC.

d) Splicing versus polyadenylation: Favored in the anti-sense direction.

e) Localization: Certain lncRNAs can occupy the chromatin, subnuclear domains, the nucleoplasm or the cytoplasm.

f) Degradation: Many unstable lncRNA transcripts are subject to the nuclear exosome or to cytosolic nonsense-mediated decay (NMD).

\section{LncRNA in different cancers}

lncRNAs have been involved in development of different cancers (Table 3).

HCC: LNCRNA AF113014 was reported to act as a tumour suppressor lncRNA that is down regulated in HCC. The tumour suppressor effect of AF113014 relies in regulation of EGR2 expression through regulation of oncogenic miRNA 20a. EGR2 belongs to EGR family and its responsible for regulation of cellular apoptosis. Oncogenic miRNA 20a acts on 3'UTR of EGR2 interfering with its expression resulting in low expression of EGR2 and so inhibition of apoptosis. In HCC low level of LNCRNA AF113014 results in upregulation of oncogenic miRNA 20a which results in low expression of EGR2 and enhancement of HCC cells proliferation and invasiveness $[62,63]$.

TP73-AS1 was found to be an oncogenic LNCRNA that is upregulated in HCC tissues and cell lines. High TP73-AS1 expression was correlated with worse clinicopathological features, poorer prognosis and shorter survival. Knockdown of TP73-AS1 inhibited the HCC proliferation, the expression levels of HMGB1, RAGE and NF$\kappa \mathrm{B}$ in HCC cells and resulted an increase in expression of mir-200a. High-mobility group box 1 protein (HMGB1) is an evolutionarily ancient and critical manager of cell death and survival. According to previous studies, the ability of proliferation, migration and invasion of HCC cells was reinforced when the expression endogenous HMGB1 was enhanced using HMGB1 DNA. HMGB1 activates RAGE signalling pathways and induces NF- $\mathrm{KB}$ activation to encourage cellular proliferation, invasion, and metastasis, in HCC cell lines. Mir 200a is an antioncogenic miRNA that is found to be downregulated in HCC Cells. Mirna- 200a expression induces inhibition of HCC cell proliferation by binding to 3'UTR of HMGB1 resulting in its downregulation and so inhibition of HMGB1 RAGE signaling pathway and induction of

\begin{tabular}{|c|c|c|}
\hline IncRNA subtype & origin & References \\
\hline $\begin{array}{c}\text { Natural Antisense } \\
\text { Transcript (NAT) }\end{array}$ & $\begin{array}{c}\text { Cis-NAT: sequence complementarity to a coding RNA at the same } \\
\text { locus } \\
\text { Trans-NAT: sequence complementarity to a coding RNA at a distal } \\
\text { genomic locus }\end{array}$ & $\begin{array}{c}\text { Regulates expression of sense partner transcript } \\
\text { Likely regulates expression of sense partner transcript, } \\
\text { may have other functions }\end{array}$ \\
\hline $\begin{array}{c}\text { Long Intergenic Noncoding } \\
\text { RNA (lincRNA) }\end{array}$ & $\begin{array}{c}\text { Encoded between known protein coding genes or within introns. } \\
\text { macroRNA or vlincRNA=very long intergenic RNA }\end{array}$ & $\begin{array}{c}\text { Functions not fully characterized, likely include } \\
\text { regulation of transcription, RNA stability, recruitment of } \\
\text { protein complexes and other subcellular elements }\end{array}$ \\
\hline Sense overlapping & Transcribed from same strand of DNA as another transcript & -- \\
\hline [59] & Originate from introns of coding genes; do not overlap with exons & [59] \\
\hline Processed Transcript & RNA transcript that is spliced or polyadenylated & [59] \\
\hline [59] \\
\hline
\end{tabular}

Table 2: Classification of IncRNAs. 
Citation: Samir A, Salama E, El-Tayebi HM (2018) The Long Non-Coding RNA XIST: A New Cornerstone in Carcinogenesis. J Mol Genet Med 12: 356 doi:10.4172/1747-0862.1000356

Page 6 of 16

\begin{tabular}{|c|c|c|c|c|}
\hline Cancer & IncRNA & Expression & Function & \\
\hline \multirow{4}{*}{$\mathrm{HCC}$} & AF113014 & Downregulated & Regulate ERG2 expression through Regulation of oncogenic mir 20a & [38] \\
\hline & TP73-AS1 & Upregulated & Affects cell proliferation through Mir-200a dependent HMGB1/RAGE regulation & [41] \\
\hline & UB32CP3 & Upregulated & Sponge for miRNA miR-138-5p & [117] \\
\hline & UC-134 & Downregulated & Inhibits HCC by inhibiting CUL4A- mediated ubiquitination of LATS1 & [42] \\
\hline \multirow{5}{*}{ NSLC } & HOXA11-AS & Upregulated & Inhibits antioncogenic mir $-200 b$ & [43] \\
\hline & linc00673 & Upregulated & Silences HOXA5 expression by recruiting EZH2 to its promotor & [44] \\
\hline & XLOC_008466 & Upregulated & ceRNA that interacts with mir-874 & [47] \\
\hline & SNHG1 & Upregulated & Targets antioncogenic mir-101-3p & [48] \\
\hline & BCAR4 & Upregulated & Promotes cell invasion by enhancing EMT & [49] \\
\hline \multirow{3}{*}{ CcRc } & MRCCAT1 & Upregulated & Negatively regulates NPR3 expression & [52] \\
\hline & NEAT1 & Upregulated & Endogenous sponge for mirna 34a & [160] \\
\hline & PANDAR & Upregulated & Regulates $\mathrm{G} 1 / \mathrm{S}$ phase transition and promotes tumor invasion & [53] \\
\hline \multirow{3}{*}{$\mathrm{CCC}$} & AFAP1-AS1 & Upregulated & Enhances cell proliferation and invasion & [54] \\
\hline & $\begin{array}{l}\text { LncRNA h19 } \\
\text { And HULC }\end{array}$ & Upregulated & Regulators of downstream inflammatory genes that initiate and sustain CCA & [56] \\
\hline & PANDAR & Upregulated & Enhances cell invasion \& inhibits apoptosis & [58] \\
\hline \multirow{6}{*}{$\begin{array}{l}\text { Breast } \\
\text { cancer }\end{array}$} & CCAT2 & Upregulated & Activates TGF Beta signaling & [59] \\
\hline & LINC00052 & Upregulated & Increases HER3 expression and HER3 signaling. & [60] \\
\hline & HOXA-AS2 & Upregulated & Endogenous sponge for mir $520 c-3 p$ & [62] \\
\hline & LIMT & Downregulated & Suppress invasiveness of cells and cell proliferation & [63] \\
\hline & LINC00628 & Downregulated & Regulates Bcl2/Bax/Caspase-3 signal pathway & [64] \\
\hline & SNHG12 & Upregulated & Upregulates MMP13 & [65] \\
\hline
\end{tabular}

Table 3: IncRNAs in different cancers.

NF-KB. TP73-AS1 executes its oncogenic function by affecting cell proliferation through miR-200a-dependent HMGB1/RAGE regulation through competing with HMGB1 for miR-200a binding resulting in inhibition of miR-200a expression, subsequently upregulated HMGB1/ RAGE expression and promotion of HCC cell proliferation [63].

LncRNA UB32CP3 acts as an oncogenic lncRNA that is highly expressed in HCC. High level of LncRNA UB32CP3 in HCC is correlated with reduced overall survival and poor prognosis. Upregulation of lncRNA UBE2CP3 significantly enhances the invasion and metastasis of HCC cells. LncRNA UBE2CP3 promotes HCC cells invasion and metastasis by inducing EMT. Furthermore, High level of UB32CP3 was correlated with decreased expression of epithelial protein marker E-cadherin, and elevated expression of mesenchymal markers N-cadherin. LncRNA UBE2CP3 was found to be located mainly in cytoplasm and it may act as a sponge for miRNA miR138-5p. LncRNA UBE2CP3 has a binding site to mir -138-5p but the relation between lncRNA UBE2CP3 and mir-138-5p is still unknown.

LncRNA uc.134 is another LncRNA mainly reported to act as antioncogenic LncRNA that is down regulated in HCC. Low expression of lncRNA uc.134 in HCC is related with poor survival of HCC patients. The antioncogenic function of LncRNA uc.134 is mediated through binding of IncRNA uc.134 to a protein called CUL4A resulting in the inhibition of its translocation from nucleus to cytoplasm, thus inhibiting cytoplasmic CUL4A mediated ubiquitination of a protein called LATS1. Ubiquitination of LATS1causes its deactivation and degradation. Unubiquitinated LATS1 in turns results in phosphorylation and inactivation of YAP a component of Hippo signalling pathway. This Phosphorylated YAP is unable to translocate from cytoplasm to nucleus, so it cannot target its downstream genes and Hippo signalling pathway becomes disrupted. Hippo signalling pathway is an evolutionary conserved pathway that controls organ size by regulating cell proliferation, apoptosis and stem cell self- renewal. Dysregulation of hippo pathway contributes to cancer development and causes promotion of cell proliferation and inhibition of cell apoptosis. Low level of uc.134 results in Hippo signalling pathway activation so enhancement of HCC cell proliferation and inhibition of apoptosis take place [64].

NSLC: The lncRNA HOXA11-AS was identified as an oncogenic lncRNA involved in NSLC. The lncRNA HOXA11-AS expression was significantly higher in NSCLC tissues compared with adjacent normal tissue. High level of IncRNA HOXA11-AS was related to poor prognosis in NSCLC patients. Furthermore, knockdown of lncRNA HOXA11-AS in NSLC cell lines resulted in inhibition of cell invasive abilities and decreased the expression of EMT related transcription factors as ZEB1, ZEB2, Snail1, Snail2 and EMT marker N-cadherin, and caused increased expression of E-cadherin. Oncogenic HOXA11-AS mechanism of action relies in promoting NSLC cell invasiveness through promoting EMT and this is mediated by inhibition of antioncogenic mir-200b. HOXA11-AS interacts with EZH2 and DNMT1 to suppress mir-200b activity. EZH2 is a gene suppressor when it becomes overexpressed, many tumor suppressor genes that are normally turned on, are turned off. DNMT1 causes hypo or hypermethylation to the genes thus altering their activity. By interacting with EZH2 and DNMT1, LncRNA HOXA11-AS mediates their recruitment to the miR-200b promoter regions which results in repression of miR-200b expression in NSCLC cells, thus promoting NSLC cell EMT and invasiveness [65]. Linc00673 is an oncogenic LncRNA that is highly expressed in NSLC. Additionally, Linc00673 was reported to be highly expressed in NSLC. High level of linc00673 promoted NSCLC cells proliferation. Linc00673 expression was positively correlated with advanced TNM stages and lymph node metastasis. Knocking -down of linc00673 results in inhibition of cancer cell migration and invasion, both in vitro and in vivo. Oncogenic LncRNA linc00673 mechanism relies in silencing of HOXA5 expression by recruiting epigenetic repressor EZH2 at its promoter regions resulting in increased metastasis of NSLC. HOXA5 is a tumour suppressor gene functioning as a transcription factor that results in inhibition of NSCLC cell metastasis by regulating cytoskeletal remodeling. 
Another LncRNA that was reported to be related to NSLC is XLOC008466. LncRNA XLOC008466 is an oncogenic LncRNA that was reported to be upregulated in NSLC. High level of LncRNA XLOC_008466 was related to lymph node metastasis and TNM stage. In vitro, down-regulation of XLOC_008466 resulted in inhibition of cell proliferation and invasion of NSLC cells and promoted cell apoptosis. LncRNA XLOC_008466 exhibits its oncogenic function through acting as a ceRNA that interacts with mir-874 and results in its inhibition. LncRNA XLOC_008466 results in downregulation of mir-874 and thus affecting mir 874 downstream targets as MMP2 and XIAP. MMP2 was found to play an important role during the invasion and metastasis of various cancers, including NSCLC XIAP can regulate the apoptosis related pathway and induces cell apoptosis in NSCLC [66,67]. Downregulated mir-874 in NSLC enhances upregulation of MMP2 and XIAP and so enhanced cell proliferation and invasiveness [68]. SNHG1 is another oncogenic LncRNA that was reported to be up-regulated in NSCLC tissues and cell lines. NSCLC patients with high SNHG1 expression were significantly correlated with larger tumour size, advanced TNM stage, lymph node metastasis and poor overall survival compared to patients with low SNHG1 expression. Inhibition of SNHG1 expression in NSCLC resulted in suppression of NSCLC cell proliferation both in vitro and in vivo. SNHG1 acts as a tumour oncogene in NSCLC by directly targeting antioncogenic mir-101-3p resulting in a decrease in its expression level. Mir-101-3p has an inhibitory effect on SOX9 gene thus abolishing role of SOX9 protein in activation of WNT/B Catenin pathway responsible for cell proliferation. In NSCLC SNHG1 directly inhibits mir-101-3p resulting in upregulation of SOX9 expression and so activation of WNT/B catenin pathway that results in cell proliferation [69]. As for BCAR4 role in NSLC, it was reported that BCAR4 is an oncogenic LncRNA that was found to be higher in NSCLC tissues than that in cancer-adjacent tissues. High level of BCAR4 was positively correlated with tumour size, clinical stage, and distant metastasis in patients with NSCLC. BCAR4 knockdown resulted in inhibition of tumour cell invasion, metastasis, proliferation, induced cell cycle arrest and increase cell apoptosis. BCAR4 oncogenic function is mediated through promoting cell invasion by enhancing EMT in NSCLC and this occurs through regulation of level of EMT molecular markers: Vimentin, N-cadherin and E-cadherin [70].

\section{Clear cell renal carcinoma}

MRCCAT1 was reported be an oncogenic LncRNA that is highly expressed in metastatic ccRCC tissues and it is associated with the metastatic properties of ccRCC. Overexpression of MRCCAT1 was correlated with promotion of ccRCC cells proliferation, migration, and invasion. Depletion of MRCCAT1 inhibited ccRCC cells proliferation, invasiveness and in vitro and in vivo migration. MRCCAT1 promotes ccRCC metastasis by negatively regulating NPR3 expression. NPR3 by being negatively joined to adenylyl cyclase and MAPK signalling pathways was previously shown to execute an important role in proliferation and metastasis of the tumor [71-73]. Adenylyl cyclase can cause stimulation of mitogen-activated protein kinase MAPK signal pathways. MRCCAT1 causes suppression of NPR3 expression by getting polycomb repressive complex 2 toward NPR3 promoter which results in repression of NPR3 gene transcription through induction of trimethylation of $\mathrm{H} 3 \mathrm{~K} 27$. This will result in activation of MAPK signalling pathway and ccRCC metastasis [74].

NEAT1 is another oncogenic LncRNA that is highly expressed in renal cell carcinoma. High level of expression of NEAT1 correlates with advanced stage, metastasis and shorter overall survival in renal cell carcinoma. Knockdown of NEAT1 suppressed RC cell growth by inhibiting cell cycle progression, invasion and migration by inhibiting EMT. Also knockdown of NEAT1 enhanced RC cells sensitivity towards chemotherapy. NEAT1 exerts its oncogenic function through acting as an endogenous sponge for antioncogenic miRNA34a resulting in decrease in its expression and enhanced expression of C-MET.

PANDAR acts as a tumour initiating gene in CCRC. PANDAR was reported to act as an oncogene in CCRC. It was reported that PANDAR is highly expressed in CCRC compared to normal tissue. Increased PANDAR was positively correlated with an advanced TNM stage. PANDAR acts as an oncogene in CCRC through regulation of G1/S phase transition and through promoting tumor invasion. Furthermore, Knockdown of PANDAR affected apoptotic protein levels the levels of Bcl-2 and Mcl-1 were downregulated, while Bax was upregulated after the silencing of PANDAR. Also knockdown of PANDAR inhibited of PI3K/AKT/mtor pathway: Phosphorylated AKT level decreased and mTOR level decreased in presence of Knocked down PANDAR [75].

Cholangiocarcinoma: AFAP1-AS1was proved to act as an oncogene in Cholangiocarcinoma and it is highly expressed in cholangiocarcinoma cells compared to normal cells. Higher AFAP1-AS1 expression was correlated with shorter overall survival. Knockdown of AFAP1-AS1 knockdown resulted in a smaller part of $S$ phase cells and an enhanced proportion of G0- G1phase cells. Also, suppression of AFAP1-AS1 impaired the expression of c-Myc and Cyclin D1. Furthermore, knockdown of AFAP1-AS1 resulted also in decreased expression of MMP-2 and MMP-9. So AFAP1-AS1 exerts its oncogenic function through enhancement of cholangiocarcinoma cell proliferation and invasion [76]. LncRNA h19 and HULC are two oncogenic LncRNA involved in cholangiocarcinoma. These two lncRNAs are expressed under oxidative stress conditions and promotes chronic inflammation of CCA cells through long term exposure of cells to oxidative stress. Interestingly, HULC and H19 exerts their oncogenic function by controlling downstream genes related to inflammation which initiate and maintain CCA. This occurs through being competing endogenous RNAs (ceRNAs) that sponge let-7a/let-7b and miR-372/miR-373 respectively. By sponging let$7 \mathrm{a} / \mathrm{let}-7 \mathrm{~b}$ and miR-372/miR-373 this will result in decrease in their expression level and increases expression of two targets for let-7a/ let-7b and miR-372/miR-373: inflammatory cytokine IL6 IL6 (one of the most vital inflammatory factors involved in the growth, migration and pathogenesis of CCA [34]. CXCR4 a chemokine receptor related to the development and metastasis of CCA respectively $[77,78]$. PANDAR is another oncogenic lncRNA in cholangiocarcinoma, high level of PANDAR in cholangiocarcinoma was correlated with lymph node metastasis, TNM stage and postoperative relapse. PANDAR exerts its oncogenic function in cholangiocarcinoma through facilitating cholangiocarcinoma cell invasion and inhibition of cholangiocarcinoma cell apoptosis. PANDAR facilitates invasion potential partly by upregulating MMP-9 expression and by enhancing EMT process. PANDAR acts as a defender against cancer apoptosis, and apoptosis-related factors (caspase-3 and caspase-9). Furthermore, knockdown of PANDAR increased expression of caspase 3 and caspase 9 and causes decrease in expression of BCL2 (anti apoptotic protein) [78].

Breast cancer: CCAT2 was identified to act as s an oncogenic lncRNA that is upregulated in BC tissue compared to normal tissue. CCAT2 level was higher in BC tissues showing metastasis than tissues with no metastasis. Knocking down of CCAT2 in BC cell lines resulted in inhibition of proliferation, migration and invasiveness of $\mathrm{BC}$ cells, 
caused BC cell cycle arrest in G0/G1 phase and induced apoptosis. In addition to a marked decrease of TGFB expression in $\mathrm{BC}$ cell lines compared to BC cells having high level of CCAT2. CCAT2 executes its oncogenic role through promoting $\mathrm{BC}$ cell proliferation, invasiveness and metastasis through induction and activation of TGFB signalling pathway [79].

LINC00052 was reported to be an oncogenic lncRNA highly expressed in BC tissue compared to normal tissue. Level of LINC00052 in BC tissue was correlated with HER3 level in BC tissue. Increased level of LINC00052 expression in BC promotes not only cell growth and survival, but also cell adhesion, spreading, and transformation of breast cancer cells. Furthermore, oncogenic function of LINC00052 in $\mathrm{BC}$ relies on increasing $\mathrm{BC}$ growth, spreading and survival and this is maintained through upregulation of HER3 expression and HER3 signalling. Also, high LINC00052 levels in BC was found to predict an increased sensitivity of breast cancer cells to HER3 neutralizing antibody treatment [80]. HOXA-AS2 is another LncRNA that is involved in BC carcinogenesis, HOXA-AS2 level is upregulated in $\mathrm{BC}$ tissues compared to normal tissue. high level of HOXA-AS2 in $\mathrm{BC}$ is correlated with clinico-pathological features. Silencing of HOXA-AS2 inhibited progression of BC cells in vivo and in vitro. Interestingly, HOXA-AS2 exerts its oncogenic function in BC by acting as an endogenous sponge for antioncogenic mir-520c-3p resulting in a decrease of mir-520c-3p expression and increase in expression of mir- $520 c-3 p$ target genes or proteins. TGF- $\beta 2$ is a member of TGF- $\beta$ family and promotes tumor metastasis. RELA is a member of the transcription factor nuclear factor kappa $\mathrm{B}(\mathrm{NF}-\mathrm{\kappa B})$ family. RELA was proved in involvement of regulation of the growth and malignancy of various tumors by regulating pro-inflammatory and pro-survival genes [81]. By acting as an endogenous sponge for mir 520c-3p, level of mir-520c-3p target proteins like TGFB2 and RELA increases in $\mathrm{BC}$ and $\mathrm{BC}$ cell invasion and growth is maintained [82]. LIMT is an antioncogenic lncRNA that was reported to be lowly expressed in $\mathrm{BC}$ tissues compared to normal tissues. Decrease in LIMT expression in $\mathrm{BC}$ tissues enhanced $\mathrm{BC}$ cells invasion, metastasis and is correlated with poorer prognosis. EGF which is highly expressed in aggressive tumors causes downregulation of LIMT expression by enhancing histone deacetylation of LIMT promoter. And so enhanced BC cells invasion and metastasis [83]. LINC00628 was also reported to be an antioncogenic LNCRNA whose level is downregulated in BC tissues compared with normal tissues. BC patients with low expressed LINC00628 had poorer prognosis and lower overall survival. The overexpression of LINC00628 resulted in suppressed breast cancer cells proliferation, invasion and migration. Also, cell cycle was arrested in G0/G1 phase in breast cancer cells and cell apoptosis was promoted as a result of LINC00628 overexpression. The expression of apoptotic proteins Caspase- 3 and Bax protein were significantly increased and the relative expression of anti- apoptotic protein $\mathrm{Bcl}-2$ protein was significantly decreased after transfection BC cell lines with LINC00628. LINC00628 exerts its anti-oncogenic function by suppressing the proliferation, invasion and migration of breast cancer cells and promotion of $\mathrm{BC}$ cell apoptosis through the regulation of $\mathrm{Bcl} 2 / \mathrm{Bax} /$ Caspase-3 signal Pathway [84]. SNHG12 is an oncogenic LncRNA highly upregulated in TNBC patients. SNHG12 was reported to be is an oncogenic LncRNA that is highly upregulated in TNBC patients. High expression of SNHG12 was positively correlated with lymph node metastasis and tumour size in TNBC patients. Additionally, SNHG12 was found to be upregulated in TNBC patients through action of C-MYC. C-MYC is considered an "amplifier" of transcription on a global scale and is always overexpressed or amplified in breast cancer
$[85,86]$. C-MYC directly interacts with SNHG12 promotor and results in upregulation of SNHG12 in TNBC tissues. MMP13 forms a part of a cluster of matrix metallo-proteinases (MMPs), and it was originally identified in breast cancer. Through degrading the extracellular matrix, MMP13 promotes tumor invasion and metastasis. A higher expression level of MMP13 is always associated with more aggressive behaviours and poorer outcome in cancers. SNHG12 was found to target directly MMP13 in TNBC and causes its upregulation in TNBC. The oncogenic role of SNHG12 relies in upregulation of MMP13 and controlling its expression level post transcriptionally in TNBC and high level of SNHG12 is maintained through C-MYC [87].

\section{XIST and TSIX}

$\mathrm{X}$-inactive specific transcript (XIST) belongs to a class of RNA molecules known as non-coding transcripts (NCT), which also includes non-coding RNAs. These ncRNAs are sometimes transcribed from DNA genes that have exons and introns; therefore, they can have various splicing isoforms and polyadenylated. It is also known as DXS399E, DXS1089, SXI1 and NCRNA00001.

The cytogenetic location is Xq13.2, which is the long (q) arm of the $\mathrm{X}$-chromosome at position 13.2 whereas the molecular location: base pairs $73,820,651$ to $73,852,753$ on the X-chromosome. XIST is located on chromosome 7. It was determined that XIST contains at least 8 exons. XIST size is 32,103 bases. XIST does not undergo posttranslational modifications. It contains repeated sequence motifs that are critical for the function [88]. Antagonizing XIST function is Tsix RNA, which is transcribed in the antisense orientation from XIST and fully overlaps with the XIST gene. Tsix is a $40-\mathrm{kb}$ RNA originating $15 \mathrm{~kb}$ downstream of Xist and transcribed across the Xist locus. Tsix sequence is conserved at the human XIC. Tsix RNA is seen exclusively in the nucleus and is localized at Xic. Before the onset of $\mathrm{X}$ inactivation, Tsix is expressed from both $\mathrm{X}$ chromosomes [89]. At the onset of $\mathrm{X}$ inactivation, Tsix expression becomes monoallelic, is associated with the future active $\mathrm{X}$ and persists until Xist is turned off. Tsix is present on Xq13.2 and the exons count is 1. Tsix is co-expressed with XIST only from the inactive X-chromosome [90]. Mammalian $\mathrm{XX}$ females equalize gene dosage relative to $\mathrm{XY}$ males by inactivation of one of their $\mathrm{X}$-chromosomes in each cell. $\mathrm{X}$ inactivation is an early developmental process that transcriptionally silences one of the pair of $\mathrm{X}$ chromosomes, thus providing dosage equivalence between males and females [91]. The process is regulated by several factors, including a region of chromosome $\mathrm{X}$ called the $\mathrm{X}$ inactivation center (XIC). The XIC comprises several non-coding and protein-coding genes, and $X I S T$ was the first non-coding gene identified within the XIC. This gene is expressed exclusively from the XIC of the inactive X chromosome and is essential for the initiation and spread of X-inactivation [91]. Initiation of $\mathrm{X}$-inactivation requires cis accumulation of large nontranslated XIST RNA, which coats the X-chromosome, followed by epigenetic changes in the future inactive chromosome. The transcript is a spliced RNA. Alternatively, spliced transcript variants have been identified, but their full-length sequences have not been determined. Mutations in the XIST promoter cause familial skewed X inactivation. Diseases associated with XIST include testicular cancer [92]. The exact molecular mechanisms by which XIST initiates $\mathrm{X}$ in activation are still under investigation whereas there are evidences that XIST plays other important roles in the differentiation, proliferation, and genome maintenance of human cells. Specifically, it has been suggested that dysfunctional expression of XIST might have a pathologic role in cancer, possibly related to changes in gene expression from alterations in the stability of heterochromatin. Although XIST typically is expressed 
by all female somatic cells, XIST expression has been found to be lost in female breast, ovarian, and cervical cancer cell lines. Meanwhile, in cell lines where XIST expression is lost, analysis of methylation of $\mathrm{X}$-linked genes reveals a loss of $\mathrm{X}$ inactivation as well. A much-debated aspect of XCI is the link between XIST spreading and recruitment of polycomb protein. The most widely accepted hypothesis predicts a direct recruitment of polycomb by XIST RNA. This interaction has been reported to be mediated by the structurally conserved XIST RepA domain, which would interact directly with polycomb repressive complex 2 (PRC2) [93]. ATRX unexpectedly functions as a highaffinity RNA-binding protein that directly interacts with RepA/Xist RNA to promote loading of PRC2. Without ATRX, PRC2 cannot load onto Xist RNA nor spread in cis along the $\mathrm{X}$ chromosome.

Moreover, epigenomic profiling reveals that genome-wide targeting of PRC2 depends on ATRX, as loss of ATRX leads to spatial redistribution of $\mathrm{PRC} 2$ and de-repression of Polycomb responsive genes. Thus, ATRX is a required specificity determinant for PRC2 targeting and function [94]. Moreover, studies demonstrated that the PRC2 cofactor Jarid2 is an important mediator of Xist-induced PRC2 targeting. The region containing the conserved B and F repeats of Xist is critical for Jarid2 recruitment via its unique $\mathrm{N}$-terminal domain. Xist-induced Jarid2 recruitment occurs chromosome-wide independently of a functional PRC2 complex, unlike at other parts of the genome, such as CG-rich regions, where Jarid2 and PRC2 binding are interdependent. Conversely, we show that Jarid2 loss prevents efficient PRC2 and H3K27me3 enrichment to Xist-coated chromatin. Jarid 2 thus represents an important intermediate between PRC2 and Xist RNA for the initial targeting of the PRC2 complex to the X chromosome during onset of XCI [93]. Additionally, a functional relationship between pluripotency and lack of XCI has been suggested, whereby pluripotency transcription factors repress the master regulator of XCI, the noncoding transcript Xist, by binding to its first intron (intron 1) [95]. Moreover, these epigenetic contexts are maintained in cancer progenitors in which SATB1 has been identified as a factor related to Xist-mediated chromosome silencing $[96,97]$. Studies showed that a matrix protein hnRNP U/SP120/SAF-A is required for the accumulation of Xist RNA on the Xi. Xist RNA and hnRNP $\mathrm{U}$ interact and upon depletion of hnRNP U, Xist RNA is detached from the $\mathrm{Xi}$ and diffusely localized into the nucleoplasm [98]. In addition, cells lacking hnRNP U expression fail to form the $\mathrm{Xi}$ so it was proposed that the association with matrix proteins is an essential step in the epigenetic regulation of gene expression by Xist RNA [98]. In mammals, loss of pluripotency and differentiation are closely linked with the onset of dosage compensation. Pluripotency factors negatively regulate Xist (the non-coding RNA that triggers $\mathrm{X}$ chromosome inactivation) and positively regulate Tsix, a repressor of Xist, to inhibit dosage compensation [99]. Interestingly, decreased Xist expression in breast cancer samples was associated with reduced levels of Jpx RNA, an lncRNA that positively regulates Xist promoter activity [100]. Rett syndrome is a neurological disorder affecting mostly girls with heterozygous mutations in the gene encoding the methyl-CpGbinding protein $(\mathrm{MeCP} 2)$ on the $\mathrm{X}$-chromosome. To identify genes involved in $\mathrm{MeCP} 2$ silencing to reverse the neurologic deficits, library of 60,000 short hair pin RNAs (shRNAs) was screened using a cell line with $\mathrm{MeCP} 2$ reporter on the Xi whereas six genes were identified in BMP/TGF-B pathway. shRNAs directed against each of these genes down-regulated XIST [101]. BRCA1, the breast and ovarian tumor suppressor was reported to regulate XIST expression, as well as colocalizes with XIST in the nucleus of female cells. Examining XIST staining in a human breast cancer cell line lacking normal BRCA1 expression showed that the loss of BRCA1 was associated with a lack of XIST staining on X inactive chromosome (Xi). When BRCA1 was reintroduced via recombinant retroviral infection, XIST staining was restored. These results suggested that BRCA1 localizes to the $\mathrm{Xi}$, where it interacts with XIST RNA [102]. Conversely, it was determined that BRCA1 may overlap in two dimensions, they actually did not co-localize with XIST when viewed in three dimensions and there was no change in XIST staining when BRCA1 was silenced using siRNAs. Moreover, the significant higher levels of XIST-RNA detected in BRCA1-associated respect to sporadic basal-like cancers, opens the possibility to use XIST expression as a marker to discriminate between the two groups of tumors. The Rnf12 gene is located approximately $500 \mathrm{~kb}$ upstream of XIST and the encoded protein stimulates XIST expression in a dosedependent manner. RNF12 expression from a single X chromosome in males is insufficient to activate Xist, whereas the double dose in females is sufficient to initiate XCI. In contrast to Xist and Tsix, RNF12 acts in Trans and activates Xist on both X chromosomes. Once the inactivation process is started on one $\mathrm{X}$ and silencing spreads over the chromosome, Rnf12 will also become silenced in cis. Studies indicate that Xist is the major downstream target of RNF12. XIST is regulated as well by miRNAs as miR-29a, miR-130a and miR-181c [103]. Loss of XIST expression is strongly correlated with up-regulation of X-linked oncogenes, accelerated growth rate in vitro, and poorer differentiation in vivo.

It was found that TSIX was overexpressed in systemic sclerosis (SSc) dermal fibroblasts both in vivo and in vitro, which was inhibited by the transfection of transforming growth factor (TGF)- $\beta 1$ siRNA. TSIX siRNA reduced the mRNA expression of type I collagen in normal and SSc dermal fibroblasts, but not the levels of major diseaserelated cytokines. In addition, TSIX siRNA significantly reduced type I collagen mRNA stability, but not protein half-lives. Furthermore, they first investigated serum lncRNA levels in patients with SSc, and serum TSIX levels were significantly increased in SSc patients. TSIX is a new regulator of collagen expression which stabilizes the collagen mRNA. The upregulation of TSIX seen in SSc fibroblasts may result from activated endogenous TGF- $\beta$ signaling and may play a role in the constitutive upregulation of collagen in these cells [104].

Histone acetyl transferases (HATs) play distinct roles in many cellular processes and are frequently mis regulated in cancers. Studies revealed that the regulatory potential of MYST1-(MOF)-containing MSL and NSL complexes in mouse embryonic stem cells (ESCs) and neuronal progenitors [105]. The MSL complex is not exclusively enriched on the $\mathrm{X}$ chromosome, yet it is crucial for mammalian $\mathrm{X}$ chromosome regulation as it specifically regulates Tsix, the major repressor of Xist lncRNA.

MSL depletion leads to decreased Tsix expression, reduced REX1 recruitment, and consequently, enhanced accumulation of $X i s t$ and variable numbers of inactivated $\mathrm{X}$ chromosomes during early differentiation [105]. The NSL complex provides additional, Tsix-independent repression of Xist by maintaining pluripotency [106]. MSL and NSL complexes therefore act synergistically by using distinct pathways to ensure a fail-safe mechanism for the repression of $\mathrm{X}$ inactivation. In the absence of Tsix, PRC2-mediated histone $\mathrm{H} 3$ lysine 27 trimethylation $(\mathrm{H} 3 \mathrm{~K} 27 \mathrm{me} 3)$ is established over the Xist promoter. Simultaneous disruption of Tsix and PRC2 leads to derepression of Xist and in turn silencing of the single X chromosome in male embryonic stem cells. Histone $\mathrm{H} 3$ lysine 36 trimethylation (H3K36me3) was identified as a modification that is recruited by Tsix co-transcriptionally and extends over the Xist promoter [107] 
Reduction of H3K36me3 by expression of a mutated histone H3.3 with a substitution of methionine for lysine at position 36 causes a significant derepression of Xist. Moreover, depletion of the H3K36 methylase Setd2 leads to upregulation of Xist, suggesting H3K36me3 as a modification that contributes to the mechanism of Tsix function in regulating XCI.

Furthermore, it was found that reduction of H3K36me3 does not facilitate an increase in $\mathrm{H} 3 \mathrm{~K} 27 \mathrm{me} 3$ over the Xist promoter, indicating that additional mechanisms exist by which Tsix blocks PRC2 recruitment to the Xist promoter. The differentially methylated DNase hypersensitive site within XIST marks the location of an alternative promoter, P2, that generates a transcript of unknown function as it lacks the A repeats that are critical for silencing. In addition, this region binds YY1 on the unmethylated inactive $\mathrm{X}$ chromosome, and depletion of YY1 untethers the XIST RNA as well as decreasing transcription of XIST [108]. Many other positive regulators of Xist can be found in the Xic region and include the non-coding RNAs Ftx and Jpx, and the pairing element Xpr. The region of the Xic upstream of Xist including Ftx, Jpx and Xpr has been shown to be enriched for H3K9 and H3K27 di- and trimethylation, respectively. These epigenetic marks may contribute to transcriptional regulation of one or more genes in the region, including Xist itself, and may result from ongoing bidirectional transcription in this region as well as from immediate recruitment of $\mathrm{PRC} 2$ by nascent $X i s t$ transcripts. The Xpr region is involved in pairing of the two X chromosomes at the onset of XCI. Deletion of Ftx in male mouse ES cells is associated with reduced transcription of Xist, Tsix and Jpx.

These findings could indicate a direct role for Ftx in Xist activation but may also be explained by Ftx-mediated global activation of the Xic region [109]. A similar role may be attributed to Jpx/Enox, which encodes another non-coding long RNA, and is located just upstream of Xist. Jpx/Enox has been shown to activate Xist in Trans, possibly by interfering with Tsix. Jpx is developmentally regulated and accumulates during XCI. Deleting Jpx blocks XCI and is female lethal. It was concluded that Xist is controlled by two RNA-based switches: Tsix for Xa and Jpx for Xi.

Other important regulators of XCI are the key pluripotency factors NANOG, OCT4, KLF4, REX1, and SOX2, and the reprogramming factor cMYC, acting as inhibitors of XCI. Binding of different combinations of these pluripotency factors at different locations in the locus can either result in repression of XIST or in activation of Tsix or Xite. NANOG, OCT4 and SOX2 bind the intron 1 region of XIST and binding of these factors has been implicated in direct repression of XIST. OCT4 is also recruited to the Tsix regulatory region and binds the Xite promoter region together with SOX2. Recruitment of these factors has been implicated in X chromosome pairing and in activation of Tsix, although binding of OCT4 and SOX2 to these specific regions has been disputed by others. OCT4 directly binds Xite and Tsix that suppress the silencer lncRNA, Xist [110]. To controls its activity as a master regulator in pluripotency and XCI, OCT4 must have chromatin protein partners. Studies showed that BRD4, a member of the BET protein subfamily, interacts with OCT4. BRD4 occupies the regulatory regions of pluripotent genes and the lncRNAs of XCI. BET inhibition or depletion of BRD4 reduces the expression of many pluripotent genes. EX1, KLF4, and $c M Y C$ are recruited to the DXPas34 region, a regulatory region in Tsix, and are involved in Tsix activation, together with YY1 and CTCF [111]. The repression of Xist is released upon differentiation, as the concentration of pluripotency factors drops, linking XCI to the pluripotent state and differentiation.
Evidence from epigenetic processes indicates that CTCF associates functionally and directly with Tsix, Xite, and Xist RNAs [112]. RS14, a conserved element that presents a strong binding site CTCF protein was investigated. Knocking out RS14 results in compromised Xist induction and aberrant XCI in female cells. Interestingly, a deletion encompassing the Xist intron 1 region that recruits OCT4, SOX2 and NANOG, only has a mild effect on XCI, leading to skewed XCI. Tsix inhibits XIST expression by several mechanisms. First, inhibition may occur by transcriptional interference [113]. Second, XIST/Tsix duplex RNA formation and processing by the RNA interference pathway may play a role by siRNA-mediated deposition of chromatin remodeling complexes. Also, recruitment of chromatin remodeling complexes by the Tsix RNA to the XIST promotor has been postulated as a possible mechanism for Tsix-mediated repression of XIST [114]. Finally, Tsix is involved in pairing of the two X-chromosomes, a process which has been implicated in initiation of XCI. Deletion of Tsix leads to upregulation of XIST and skewed XCI with preferential inactivation. Impaired transcription of Tsix has also been reported to lead to ectopic $\mathrm{XCI}$ in male cells so that Xist and Tsix are the master regulatory switch genes in XCI. Interestingly, in female cells with a deletion in Xite, a positive regulator of Tsix located upstream of Tsix, XCI is still initiated on the X-chromosome [115]. This suggests that activation of XIST is regulated by other factors. The dynamic relationship between Xist and Tsix in female cells is as follows: (i) before the onset of $\mathrm{X}$ inactivation, Xist and Tsix are biallelically co-expressed; (ii) at the onset of $\mathrm{X}$ inactivation (differentiation), Tsix becomes monoallelic, preceding upregulation of Xist RNA on the future inactive X (species 2); (iii) in the monoallelic form, Tsix RNA associates only with the future active $\mathrm{X}$; and (iv) once $\mathrm{X}$ inactivation is established, Tsix is repressed.

Tsix expression is specific to undifferentiated cells regardless of sex, persists briefly at the onset of $\mathrm{X}$ inactivation, appears to associate only with the future active $\mathrm{X}$ and disappears after $\mathrm{X}$ inactivation is established. This profile suggests that Tsix may regulate events at the onset of $\mathrm{X}$ inactivation. Its antisense nature raises the possibility that Tsix directly blocks Xist RNA action on the X chromosome [116].

$X I S T$ regulation and oncotherapy: Recently, there is a growing interest towards understanding the relation between lncRNAs and the therapeutic outcomes of cancer patients including chemotherapeutic resistance, disease-free periods and survival rates. A study suggested that overexpression of XIST in NSCLC resulted in poor prognosis due to the development of Cisplatin resistance [117]. Another study demonstrated that high levels of XIST in cervical squamous cell carcinoma is associated with high survival rates while treatment with chemo and radiotherapy [118]. In BRCA1 Breast cancer, overexpression of XIST resulted in poor outcome after treatment with high dose-alkylating agent [119].

Another study revealed that XIST levels correlated significantly with taxol sensitivity, however, XIST showed no significance correlation with Carboplatin in ovarian cancer [120]. Although XIST responsible for Cisplatin resistance in human lung adenocarcinoma which leads to worse prognosis [121]. Moreover, knockdown of XIST increase sensitivity to temozolomide alkylating agent in glioma cells [122]. Finally, XIST knockdown resulted in increase the sensitivity of nasopharangeal carcinoma cells to radiotherapy through manipulation of miR-29c [123].

\section{Role of XIST in cancer}

XIST is involved in development of many cancers as well as its ability to act as a biomarker (Tables 4 and 5). 


\begin{tabular}{|c|c|c|c|}
\hline Cancer type & XIST expression & Function & References \\
\hline Colorectal cancer & Overexpressed & Promotes cell proliferation through the inhibition of mirna1323p & [92] \\
\hline Esophegal cancer & Overexpressed & Acts as molecular sponge for mir-101 \& increases $\quad E Z H 2$ exp. & [119] \\
\hline Lung cancer & Overexpressed & Promotes cell growth through regulating mir -140 & [93] \\
\hline Non-small lung cancer & Overexpressed & $\begin{array}{l}\text { Inhibits mir-186-5p. } \\
\text { ceRNA that attenuates function of mir }-449 a \text {. } \\
\text { Interacts with EZH2 \& supress the expression of KFL2 }\end{array}$ & $\begin{array}{l}{[94]} \\
{[96]} \\
{[97]}\end{array}$ \\
\hline Osteosarcoma & Overexpressed & Acts as an endogenous sponge of mir-320b & [98] \\
\hline \multirow{3}{*}{$\mathrm{HCC}$} & \multirow{2}{*}{ Low expressed } & Low expressed due to inhibitory effect of mir- 181 & [99] \\
\hline & & Low Expressed due to inhibitory effect of mir-92 & {$[100]$} \\
\hline & Overexpressed & Acts as an endogenous sponge for mir- $139-5 p$ & [101] \\
\hline Pancreatic cancer & Overexpressed & Acts as endogenous sponge for mir $-133 a$ & [102] [103] \\
\hline \multirow{2}{*}{ Gastric cancer } & \multirow{2}{*}{ Overexpressed } & Acts as an endogenous sponge for antioncogenic mir-497 & {$[105]$} \\
\hline & & Acts as An endogenous sponge for mir- 101 & {$[106]$} \\
\hline Nasopharyngeal cancer & Overexpressed & Acts as endogenous sponge for antioncogenic miR-34a-5p & {$[109]$} \\
\hline Breast cancer & Low expressed & Its downregulation enhances AKT pathway activation & {$[110]$} \\
\hline Glioblastoma & Overexpressed & Inhibits mirna 152 & [114] \\
\hline
\end{tabular}

Table 4: XIST role in different cancers.

\begin{tabular}{|l|l|l|}
\hline Cancer Type & XIST as biomarker & References \\
\hline Ovarian cancer & $\begin{array}{l}\text { Reduction of the expression of XIST can decrease the sensitivity of OC cells to paclitaxel Suggesting that XIST can act as } \\
\text { biomarker for ovarian cancer prognosis }\end{array}$ & [108] \\
\hline \multirow{3}{*}{ Breast cancer } & $\begin{array}{l}\text { XIST expression can be used as a biomarker in BRCA1 mutated BC patients to Determine if they will benefit from HD } \\
\text { therapy than conventional therapy }\end{array}$ & [111] \\
\cline { 2 - 3 } & XIST can be used as a biomarker to predict response of BC patients towards Abexinostat \\
\hline Testicular cancer & Presence of unmethylated XIST can act as biomarker for testicular cancer & [121] \\
\hline Prostate cancer & Hypomethylated XIST can acts as a biomarker for aggressive prostate cancers. & [115] \\
\hline
\end{tabular}

Table 5: XIST as biomarker in different cancers.

Colorectal cancer: XIST was found to act as an oncogene in human colorectal cancer. The expression level of XIST was significantly high in CRC tissues sample and cells. XIST promoted CRC cell proliferation by affecting the cell cycle, through direct Inhibition of mir-132-3p resulting in MAPK1 activation. Mir -132-3p in turn acts as an antioncogene through inhibition of MAPK1 as MAPK1 is a direct target spot of miR-132-3p [124].

Oesophageal cancer: XIST was proved to have an oncogene role in oesophageal cancer. XIST was overexpressed in tumour tissues compared with that in normal counterparts. Additionally, High level of XIST was correlated with poor prognosis. Silencing of XIST expression resulted in inhibition of proliferation, migration and invasion capacity of ESCC cells in vitro and suppressed tumour growth in vivo. Furthermore, XIST is an oncogene in oesophageal cancer by acting as a molecular sponge for mir-101 and increasing EZH2 expression. XIST competes with mir-101 on its 3' UTR binding site present on EZH2 resulting in an increase in EZH2 expression. EZH2 expression increases and becomes overexpressed in ESCC compared to normal tissue and its overexpression is correlated with poorer prognosis.

Lung cancer: In lung cancer XIST plays indeed an important oncogenic role. XIST was found to be upregulated in lung cancer tissue and cell lines. High level of XIST expression promoted lung cancer cell growth. Knockdown of XIST inhibited the proliferation and promoted cell apoptosis of human LCC and suppressed metastasis in vitro and in vivo.

Interestingly, XIST acts as an oncogene in lung cancer through promotion of lung cancer cell growth by targeting miR-140 and regulating miR-140-dependent inhibitor of apoptosis-stimulating protein of p53 (iASPP) [125].
Non-small lung cancer: XIST was found to be upregulated in NSCLC tissues compared to normal tissue. XIST acts as an oncogene in NSCLC through inhibition of expression of mir-186-5p. Mir-186-5p acts as tumor suppressor miRNA in NSCLC, as mir 186-5p inhibition enhances cell proliferation and invasiveness. Furthermore, miR-186$5 p$ overexpression has been shown to block the oncogenic effects of XIST on cell proliferation and invasion [126].

High XIST level in NSLC was also reported to be linked to shorter survival and poorer prognosis. It was reported in a study that XIST can act as an oncogene through interacting with EZH2 to suppress transcription of its potential target KLF2. XIST oncogenic functions partly depend on silencing KLF2 expression. And the high level of XIST expression is corelated with a poor NSLC patient prognosis [127].

XIST was also proved to be uses as a biomarker in NSCLC patient's serum. It was reported in a study that XIST and another LncRNA called HIF1A-AS1 levels were upregulated in NSCLC tissues compared to normal tissues. Also, their level was high in serum of NSCLC patients than in healthy serums. There was a correlation between levels of XIST and HIF1A in tissues and their level in serum. Additionally, Preoperative level of XIST and HIF1A level in NSCLC serums was different than post-operative level. ROC curves for level of XIST and HIF1A in serum showed strong separation between the NSCLC patients and control group. Furthermore, combining measurement of XIST HIF1AAS1 in NSCLC patients serum yielded an AUC of 0.931 (95\% CI: $0.869-0.990 ; \mathrm{P}<0.001)$, which was significantly improved as compared to XIST (AUC $=0.834)$ or HIF1A-AS1 (AUC=0.876) alone [128].

Osteosarcoma: XIST was reported to be upregulated in osteosarcoma cells compared to normal cells. High level of XIST increased OS cell proliferation and invasiveness. XIST acts as an oncogene in osteosarcoma by acting as an endogenous sponge of mir- 
$320 \mathrm{~b}$ resulting in repression of mir-320b expression and increased expression of one of mir-320b target proteins: RAP2B which results in promotion of OS cells proliferation and migration [129].

HCC: XIST role in HCC remains elusive as studies done to discover its role in HCC are contradicting. A study has reported that: XIST acts as anti-oncogenic LncRNA in HCC. XIST expression in HCC tissues and cells is downregulated compared to normal tissues. XIST expression is abolished in HCC due to oncogenic effect of mir-181a. Mir-181a acts as oncogenic miRNA in HCC that inhibits XIST expression by acting on its multiple binding sites present on XIST LncRNA. Mir-181a results in a decrease of XIST expression in HCC and also inhibits PTEN expression. The loss of the PTEN will result in activation of PI3K/AKT signalling pathway and promotion of HCC metastasis [130].

Another study has also proved XIST role as antioncogenic in HCC. The study results have proved that Mir-92b is an oncogenic miRNA that was found to be upregulated in HCC. MiR-92b expression was significantly correlated with gender and microvascular invasion of HCC. Also, plasma level of mir-92b was high in HCC patients than in normal patients. Mir-92b oncogenic function resides in induction of B catenin signalling pathway and promotion of HCC proliferation and metastasis and this is mediated by inhibition of SMAD7 protein. $S m a d 7$ is a potential inhibitor of TGF- $\beta / \beta$-catenin signalling pathway and it is downregulated in HCC tissues compared to normal tissue. Furthermore, Mir-92b inhibits XIST expression in HCC patients. Knocking down of XIST induced upregulation of miR-92b and promotion of HCC cell proliferation and metastasis [131].

However, another study has denied XIST function as an oncogene in HCC. The study reported that XIST was overexpressed in HCC tissues compared to normal tissue. XIST expression was correlated with tumour size, knocking down of XIST decreased HCC cells proliferation both in vivo and in vitro. Additionally, XIST promoted cell cycle progression from $\mathrm{G} 1$ to $S$ phase and protected HCC cells from apoptosis. XIST exerts its oncogenic function in HCC through acting as an endogenous sponge for anti-oncogenic mir-139-5p. XIST supress mir-139-5p expression in HCC and increases expression of a potential target for mir-139-5p: PDK1. PDK1 can activate AKT kinase on the activation loop at T308, which results in promotion of full activation of AKT. Activation of PDK1 results in upregulation of cyclin D1 during cell cycle progression from G0-G1 to S phase. PDK1 is a transducer of PI3K signalling and activates multiple downstream effectors. XIST positively regulates PDK1 expression through miR-139-5p inhibition and promotes HCC cell growth by regulating the AKT signalling through miR-139-5p/PDK1 axis [132].

Pancreatic cancer: XIST was reported to be an oncogenic lncRNA that is upregulated in PC tissues than in normal tissues. Higher XIST expression was observed more often in patients with larger tumour size and advanced TNM stage. XIST exerts its oncogenic property in PC by enhancing PC cells proliferation through acting as an endogenous sponge for anti-oncogenic mir -133a and by competing with EGFR for mir-133a binding. EGFR is a transmembrane glycoprotein overexpressed in PC (1) The phosphorylation of EGFR activates downstream signalling cascade, like MAPK, PI3 K/Akt, and Src pathways, which have been involved in carcinogenesis by affecting cell proliferation, survival, invasion, and metastasis (2) Additionally, EGFR over-expression is related to poor survival, correlating with a more advanced stage, and the presence of metastases in PC. XIST by acting as an endogenous sponge for mir-133a results in decrease expression of mir-133a and through competing with EGFR for mir-133a results in inability of mir-133a to inhibit EGFR expression and so higher level of EGFR and promotion of PC Cell proliferation [133-135].
Gastric cancer: XIST was reported to act as an oncogene in gastric cancer and which level was up-regulated in GC patient tissues and cell lines compared to normal tissues. High XIST expression was significantly correlated with tumour size, lymph node metastasis and late TNM stage. XIST knockdown decreased GC cells proliferation and invasiveness both in vivo and in vitro. XIST promoted GC cell cycle progression from the G1 phase to the S phase. Additionally, XIST may protect GC cells from apoptosis. Interestingly, XIST exerts its oncogenic property through acting as an endogenous sponge for anti-oncogenic mir-497 resulting in inhibition of mir-497 in GC cells and increases MACC1 expression. MACC1 is a regulator of $\mathrm{HGF} / \mathrm{c}$ Met signalling, that triggers various malignant behaviours in GC [27]. Its expression correlates positively with GC progression, and it is considered to be an oncogene. Deregulation of MACC1 was reported to promote cell proliferation and invasion in GC cells. By increasing MACC1 expression and decreasing mir-497 expression XIST succeeds in enhancement of GC cell proliferation [136].

In another study, it reported that XIST can exerts its oncogenic function through enhancement of GC cell proliferation and this is mediated by acting as endogenous sponge for anti-oncogenic miRNA-101 resulting in a decrease in mir-101 expression in GC patients and increase in one of mir-101 targets EZH2 expression [137].

Ovarian cancer: It was reported in a study that, XIST was not detected in more than half of the OC cells with Barr body staining compared with normal ovarian cells. Other studies also showed that reduction of the expression of XIST can decrease the sensitivity of OC cells to paclitaxel, suggesting that XIST can serve as a biomarker of the prognosis of OC [138]

Nasopharyngeal cancer: XIST was reported to be an oncogenic lncRNA that is up-regulated in NPC tissues High expression of XIST contributed to a poorer survival time. Additionally, XIST over-expression enhanced nasopharyngeal carcinoma cell growth. Furthermore, XIST exerts its oncogenic function through acting as a competitive endogenous sponge for anti-oncogenic miR-34a-5p resulting in decreased expression of mir-34a-5p and up regulation of the expression of miR-34a-5p targeted gene E2F3 [139].

Breast cancer: XIST in breast cancer was reported to act as a tumor suppressor lncRNA as well as acting as a biomarker in BC patients to determine their response to high dose alkylating chemotherapy and the histone deacetylase inhibitor Abexinostat.

XIST was proved to act as a tumour suppressor lncRNA in BC, whose level was downregulated in BC tissues. XIST downregulation in $B C$ resulted in enhancement of $B C$ cell viability through enhancement of AKT phosphorylation which will result in activation of AKT pathway. AKT phosphorylation was a consequence of downregulated SPEN. SPEN is a protein important in the maintenance of XCI and XIST function. Upon XIST downregulation, SPEN is also downregulated. Downregulated SPEN results in inability of sequestering of HDAC3 (histone deacetylase 3) near X chromosome and so enhancement of HDAC3 binding to PHPPL1 whose function is to act as a phosphatase that dephosphorylates AKT. By binding to PHPPL1, HDAC3 causes deacetylation in promoter of PHPPL1 resulting in decrease of PHPPL1 expression and inability to dephosphorylate AKT and so high level of phosphorylated AKT, activated AKT pathway and enhanced BC cell viability [58].

As for XIST function as a biomarker for BC patients response to different therapies, it was reported in a study that measuring level of $X I S T$ and 53BP1 can be used as a biomarker in BRCA1 like breast 
cancer patients in prediction of $\mathrm{BC}$ patient outcome and resistance or responsiveness to high dose alkylating chemotherapy. It was found that patients with BRCA1 mutation having low XIST and high 53BP1 showed better response to HD chemotherapy rather than conventional therapy. On the contrary patients with BRCA1 mutation having high XIST and low 53BP1 showed bad response to HD chemotherapy rather than conventional therapy [119].

Another Study has reported that XIST level can be used as a biomarker to predict response of BC patients towards treatment with histone deacetylase inhibitor: Abexinostat. It was proved in this study that breast cancer cell lines having low expression of XIST can benefit from Abexinostat and displayed low population of Cancer Stem Cells. On the contrary cancer cell lines having high level of XIST did not benefit from Abexinostat treatment and CSC population increases $[121,140]$.

Glioblastoma: XIST was reported to act as an oncogene in human glioblastoma cells. XIST level is upregulated in Human glioblastoma compared to normal cells. Furthermore, Knockdown of XIST decreased human glioblastoma cell proliferation, invasiveness and cell growth. XIST exerts its oncogenic function through inhibition of mir-152 in Human glioblastoma [141].

Testicular cancer: It was proved in a study that unmethylated XIST can serve as a biomarker for presence of testicular cancer. The study reported that in normal somatic male cells XIST showed complete methylation through the $\mathrm{CpG}$ sites, including the minimum promoter and XIST-conserved repeats However in testicular cancer cells. The 5 end of the XIST gene is mainly hypomethylated [142].

Prostate cancer: XIST was found to be partially hypomethylated in prostate cancer tissues than in normal tissues. Furthermore, level of XIST in prostate cancer tissues was slightly increased than in normal tissues. Hypomethylated XIST was able to act as a biomarker for aggressive prostate cancers [143].

TSIX in cancers: As for TSIX which antagonises XIST function, its contribution and involvement in carcinogenesis is barely revealed. A single study has stated that level of TSIX in BC was found to be down regulated however its role in $\mathrm{BC}$ is still unrevealed [144-173].

\section{Conclusion}

This review has been based on summarizing the definition of epigenetics and it's types including DNA methylation, histone modification, chromatin modification and most importantly noncoding RNAs which are a recent group of RNAs that contribute in various regulatory processes. LncRNAs are ncRNAs which undergo numerous functions including disease progression. They are believed to play a pivotal role in carcinogenesis and pathogenesis of different diseases. Among important lncRNAs are XIST which play a profound role in a process known as $\mathrm{X}$-chromosome inactivation in females to achieve dosage compensation between males and females during embryonic development. TSIX is the anti-sense of XIST that had no definite role in cancers; nevertheless, being the anti-sense of XIST raised the urge to review about it. Interestingly, several studies included suggesting the strong correlation between XIST expression levels and chemotherapeutic responses. Most importantly, lncRNAs were proved to serve as a prognostic biomarker for chemotherapeutic agents in many cancers.

\section{References}

1. Deans C, Maggert KA (2015) What do you mean, "epigenetic"? Genetics 199: 887-896.
2. Kraft $P$, Horvath $S$ (2003) The genetics of gene expression and gene mapping Trends Biotechnol 21: 377-378

3. Feinberg AP (2013) The epigenetic basis of common human disease. Trans Am Clin Climatol Assoc 124: 84-93.

4. Portela A, Esteller M (2010) Epigenetic modifications and human disease. Nat Biotechnol 28: 1057-1068.

5. Hughes T, Sawalha AH (2011) The role of epigenetic variation in the pathogenesis of systemic lupus erythematosus. Arthritis Res Ther 13: 245

6. Gos M (2013) Epigenetic mechanisms of gene expression regulation in neurological diseases. Acta Neurobiol Exp (Wars) 73: 19-37.

7. Ptak C, Petronis A (2010) Epigenetic approaches to psychiatric disorders. Dialogues Clin Neurosci 12: 25-35.

8. Jaenisch R, Bird A (2003) Epigenetic regulation of gene expression: how the genome integrates intrinsic and environmental signals. Nat Genet 33: 245-54.

9. Li S (2016) DNA methylation variation trends during the embryonic development of chicken. PLoS One 11: e0159230.

10. Handy DE, Castro R, Loscalzo J (2011) Epigenetic modifications: Basic mechanisms and role in cardiovascular disease. Circulation 12: 2145-2156.

11. Singh RS, Choudhary M, David JR (1987) Contrasting patterns of geographic variation in the cosmopolitan sibling species Drosophila melanogaster and Drosophila simulans. Biochem Genet 25: 27-40.

12. Jin B, Li Y, Robertson KD (2011) DNA methylation: Superior or subordinate in the epigenetic hierarchy? Genes Cancer 2: 607-617.

13. Liao J (2015) Targeted disruption of DNMT1, DNMT3A and DNMT3B in human embryonic stem cells. Nat Genet 47: 469-78.

14. Klug M (2013) 5-Hydroxymethylcytosine is an essential intermediate of active DNA demethylation processes in primary human monocytes. Genome Biol 14: R46.

15. Estecio MR, Issa JP (2011) Dissecting DNA hypermethylation in cancer. FEBS Lett 585: 2078-2086.

16. Ehrlich M (2009) DNA hypomethylation in cancer cells. Epigenomics 1: 239-259.

17. Kulis M, Esteller M (2010) DNA methylation and cancer. Adv Genet 70: 27-56.

18. Bannister AJ, Kouzarides T (2011) Regulation of chromatin by histone modifications. Cell Res 21: 381-95.

19. Eberharter A, Becker PB (2002) Histone acetylation: A switch between repressive and permissive chromatin. Second in review series on chromatin dynamics. EMBO Rep 3: 224-229.

20. West AC, Johnstone RW (2014) New and emerging HDAC inhibitors for cancer treatment. J Clin Invest 124: 30-39.

21. Greer EL, Shi Y (2012) Histone methylation: A dynamic mark in health, disease and inheritance. Nat Rev Genet 13: 343-357.

22. Huang $Y$ (2012) Inhibitors of histone demethylation and histone deacetylation cooperate in regulating gene expression and inhibiting growth in human breas cancer cells. Breast Cancer Res Treat 131: 777-789.

23. Peterson CL, Laniel MA (2004) Histones and histone modifications. Curr Bio 14: R546-551.

24. Berger SL (2002) Histone modifications in transcriptional regulation. Curr Opin Genet Dev 12: 142-148.

25. Geiman TM, Robertson KD (2002) Chromatin remodeling, histone modifications and DNA methylation-how does it all fit together? J Cell Biochem 87: 117-125.

26. Liu B, Yip R, Zhou Z (2012) Chromatin remodeling, DNA damage repair and aging. Curr Genomics 13: 533-547.

27. Lasserre FD, Villagra CA (2017) The importance of ncRNAs as epigenetic mechanisms in phenotypic variation and organic evolution. Front Microbiol 8: 2483.

28. Andersen AA, Panning B (2003) Epigenetic gene regulation by noncoding RNAs. Curr Opin Cell Biols 15: 281-289.

29. Song MS, Rossi JJ (2017) Molecular mechanisms of Dicer: endonuclease and enzymatic activity. Biochem J 474: 1603-1618.

30. Carthew RW, Sontheimer EJ (2009) Origins and mechanisms of miRNAs and siRNAs. Cell 136: 642-655. 
Citation: Samir A, Salama E, El-Tayebi HM (2018) The Long Non-Coding RNA XIST: A New Cornerstone in Carcinogenesis. J Mol Genet Med 12: 356 doi:10.4172/1747-0862.1000356

Page 14 of 16

31. Mattick JS, Makunin IV (2006) Non-coding RNA. Hum Mol Genet 15: R17-29.

32. Rinn JL, Chang HY (2012) Genome regulation by long noncoding RNAs. Annu Rev Biochem 81: 145-166.

33. Matsui M, Corey DR (2017) Non-coding RNAs as drug targets. Nat Rev Drug Discov 16: 167-179.

34. Costa FF (2005) Non-coding RNAs: New players in eukaryotic biology Gene357: 83-94.

35. Stein LD (2004) Human genome: End of the beginning. Nature 431: 915-916.

36. Taft RJ (2010) Non-coding RNAs: Regulators of disease. J Pathol 220: 126-39.

37. Hock J, Meister G (2008) The argonaute protein family. Genome Biol 9: 210.

38. Meister G, Tuschl T (2004) Mechanisms of gene silencing by double-stranded RNA. Nature 431: 343-349.

39. Lippman Z, Martienssen R (2004) The role of RNA interference in heterochromatic silencing. Nature 431: 364-370.

40. Houwing S (2007) A role for Piwi and piRNAs in germ cell maintenance and transposon silencing in Zebrafish. Cell 129: 69-82.

41. Griffiths-Jones S (2006) miRBase: The microRNA sequence database. Methods Mol Biol 342: 129-138.

42. Krol J, Loedige I, Filipowicz W (2010) The widespread regulation of microRNA biogenesis, function and decay. Nat Rev Genet 11: 597-610.

43. Grey $F$ (2010) A viral microRNA down-regulates multiple cell cycle genes through mRNA 5'UTRs. PLoS Pathog 6: e1000967.

44. Orom UA, Nielsen FC, Lund AH (2008) MicroRNA-10a binds the 5'UTR of ribosomal protein mRNAs and enhances their translation. Mol Cell 30: 460-471.

45. Lim LP (2005) Microarray analysis shows that some microRNAs downregulate large numbers of target mRNAs. Nature 433: 769-773.

46. Li M (2010) microRNA and cancer. AAPS J 12: 309-317.

47. Kiss T (2002) Small nucleolar RNAs: an abundant group of noncoding RNAs with diverse cellular functions. Cell 109: 145-148.

48. Vidovic I (2000) Crystal structure of the spliceosomal $15.5 \mathrm{kD}$ protein bound to a U4 snRNA fragment. Mol Cell 6: 1331-1342.

49. Costa FF (2010) Non-coding RNAs: Meet thy masters. Bioessays 32: 599-608.

50. Morris KV (2008) Bidirectional transcription directs both transcriptional gene activation and suppression in human cells. PLoS Genet 4: e1000258.

51. Janowski BA, Corey D (2010) Minireview: Switching on progesterone receptor expression with duplex RNA. Mol Endocrinol 24: 2243-2252.

52. Preker P (2008) RNA exosome depletion reveals transcription upstream of active human promoters. Science 322: 1851-1854.

53. Khawar (2018) Multifactorial role of long non-coding RNAs (LncRNAs) in hematopoiesis. Front Biosci 10: 119-126.

54. Espinosa JM (2017) On the origin of IncRNAs: Missing link found. Trends Genet 33: 660-662

55. Mathy NW, Chen XM (2017) Long non-coding RNAs (IncRNAs) and their transcriptional control of inflammatory responses. J Biol Chem 292: 1237512382.

56. Fang $Y$, Fullwood MJ (2016) Roles, functions, and mechanisms of long noncoding RNAs in cancer. Genomics Proteomics Bioinformatics 14: 42-54.

57. Loos $F(2016)$ Xist and Tsix transcription dynamics is regulated by the X-toautosome ratio and semistable transcriptional states. Mol Cell Biol 36: 2656-2667.

58. Huang YS (2016) Xist reduction in breast cancer upregulates AKT phosphorylation via HDAC3-mediated repression of PHLPP1 expression. Oncotarget 7: 43256-43266.

59. Peschansky VJ, Wahlested C (2014) Non-coding RNAs as direct and indirect modulators of epigenetic regulation. Epigenetics, 2014. 9: 3-12.

60. Kung JT, Colognori D, Lee JT (2013) Long noncoding RNAs: past, present, and future. Genetics 193: 651-669.

61. Soudyab M, Iranpour M, Ghafouri-Fard S (2016) The Role of long non-coding RNAs in breast cancer. Arch Iran Med 19: 508-517.
62. Zeng T (2017) LncRNA-AF113014 promotes the expression of Egr2 by interaction with miR-20a to inhibit proliferation of hepatocellular carcinoma cells. PLoS One 12: e0177843.

63. Li S (2017) The long non-coding RNA TP73-AS1 modulates HCC cell proliferation through miR-200a-dependent HMGB1/RAGE regulation. J Exp Clin Cancer Res 36: 51.

64. Ni W (2017) A novel IncRNA uc.134 represses hepatocellular carcinoma progression by inhibiting CUL4A-mediated ubiquitination of LATS1. J Hematol Oncol 10: 91.

65. Chen JH (2017) Overexpression of IncRNA HOXA11-AS promotes cel epithelial-mesenchymal transition by repressing miR-200b in non-small cell lung cancer. Cancer Cell Int 17: 64.

66. Wang H (2013) miRNA-29c suppresses lung cancer cell adhesion to extracellular matrix and metastasis by targeting integrin beta1 and matrix metalloproteinase2 (MMP2). PLoS One 8: e70192.

67. Qin S (2016) XIAP inhibits mature Smac-induced apoptosis by degrading it through ubiquitination in NSCLC. Int J Oncol 49: 1289-1296.

68. Yang R (2017) Long non-coding RNA XLOC_008466 functions as an oncogene in human non-small cell lung cancer by targeting miR-874. Cell Physio Biochem 42: 126-136.

69. Cui $Y$ (2017) Upregulated IncRNA SNHG1 contributes to progression of nonsmall cell lung cancer through inhibition of miR-101-3p and activation of Wnt/ beta-catenin signaling pathway. Oncotarget 8: 17785-17794.

70. Li N, Gao WJ, Liu NS (2017) LncRNA BCAR4 promotes proliferation, invasion and metastasis of non-small cell lung cancer cells by affecting epithelialmesenchymal transition. Eur Rev Med Pharmacol Sci 21: 2075-2086.

71. Lelievre V (2001) Proliferative actions of natriuretic peptides on neuroblastoma cells. Involvement of guanylyl cyclase and non-guanylyl cyclase pathways. J Biol Chem 276: 43668-43676.

72. Sellitti DF (2001) Natriuretic peptides increase cAMP production in human thyrocytes via the natriuretic peptide clearance receptor (NPR-C). Regul Pept 97: 103-109.

73. Li JK (2017) Long non-coding RNA MRCCAT1 promotes metastasis of clear cell renal cell carcinoma via inhibiting NPR3 and activating p38-MAPK signaling. Mol Cancer 16: 111.

74. Xu Y (2017) An increase in long non-coding RNA PANDAR is associated with poor prognosis in clear cell renal cell carcinoma. BMC Cancer 17: 373.

75. Lu X (2017) Long noncoding RNA AFAP1-AS1 promoted tumor growth and invasion in cholangiocarcinoma. Cell Physiol Biochem 42: 222-230.

76. Zhao S, Wang J, Qin C (2014) Blockade of CXCL12/CXCR4 signaling inhibits intrahepatic cholangiocarcinoma progression and metastasis via inactivation of canonical Wnt pathway. J Exp Clin Cancer Res 33: 103.

77. Wang WT (2016) LncRNAs H19 and HULC, activated by oxidative stress, promote cell migration and invasion in cholangiocarcinoma through a ceRNA manner. J Hematol Oncol 9: 117.

78. Xu Y, Jiang X, Cui Y (2017) Upregulated long noncoding RNA PANDAR predicts an unfavorable prognosis and promotes tumorigenesis in cholangiocarcinoma. Onco Targets Ther 10: 2873-2883.

79. Wu ZJ 2017) Long non-coding RNA CCAT2 promotes the breast cancer growth and metastasis by regulating TGF-beta signaling pathway. Eur Rev Med Pharmacol Sci 21: 706-714.

80. Salameh A (2017) HER3 and LINC00052 interplay promotes tumor growth in breast cancer. Oncotarget 8: 6526-6539.

81. Kasashima $H$ (2017) Clinicopathologic significance of the CXCL1-CXCR2 axis in the tumor microenvironment of gastric carcinoma. PLoS One 12: e0178635.

82. Fang Y (2017) Long non-coding RNA HOXA-AS2 promotes proliferation and invasion of breast cancer by acting as a miR-520c-3p sponge. Oncotarget 8 : 46090-46103.

83. Chen SA (2016) LIMT is a novel metastasis inhibiting IncRNA suppressed by EGF and downregulated in aggressive breast cancer. EMBO Mol Med 8: 1052-1064.

84. Chen DQ (2017) Long non-coding RNA LINC00628 suppresses the growth and metastasis and promotes cell apoptosis in breast cancer. Eur Rev Med Pharmacol Sci 21: 275-283. 
85. Dang CV (2012) MYC on the path to cancer. Cell 149: 22-35.

86. Cancer Genome Atlas N (2012) Comprehensive molecular portraits of human breast tumours. Nature 490: 61-70.

87. Lago SJ (2017) Erratum to: Cancer progression by breast tumors with Pit-1 overexpression is blocked by inhibition of metalloproteinase (MMP)-13. Breast Cancer Res 19: 38.

88. Brockdorff N, Duthie SM (1998) X chromosome inactivation and the Xist gene. Cell Mol Life Sci 54: 104-112.

89. Gayen S (2015) A primary role for the Tsix IncRNA in maintaining random X-chromosome inactivation. Cell Rep 11: 1251-1265.

90. Lee JT, Davidow LS, Warshawsky D (1999) Tsix, a gene antisense to Xist at the X-inactivation centre. Nat Genet 21: 400-404.

91. Xie $P(2016)$ The dynamic changes of $X$ chromosome inactivation during early culture of human embryonic stem cells. Stem Cell Res 17: 84-92.

92. Looijenga LH (1997) X inactivation in human testicular tumors. XIST expression and androgen receptor methylation status. Am J Pathol 15: 581-590.

93. Rocha DA (2014) Jarid2 Is Implicated in the Initial Xist-Induced Targeting of PRC2 to the Inactive $X$ chromosome. Mol Cell 53: 301-316.

94. Sarma K (2014) ATRX directs binding of PRC2 to Xist RNA and Polycomb targets. Cell 159: 869-883.

95. Minkovsky A (2013) The pluripotency factor-bound intron 1 of Xist is dispensable for $\mathrm{X}$ chromosome inactivation and reactivation in vitro and in vivo. Cell Rep 3 : 905-918.

96. Agrelo R (2011) X inactivation and progenitor cancer cells. Cancers (Basel) 3 2169-2175.

97. Agrelo R (2009) SATB1 defines the developmental context for gene silencing by Xist in lymphoma and embryonic cells. Dev Cell 16: 507-516.

98. Hasegawa $Y$ (2010) The matrix protein hnRNP $U$ is required for chromosomal localization of Xist RNA. Dev Cell 19: 469-476.

99. Shibata S, Lee JT (2010) Characterization and quantitation of differential Tsix transcripts: implications for Tsix function. Hum Mol Genet 12: 125-36.

100. Tian D, Sun S, Lee JT (2010) The long noncoding RNA, Jpx, is a molecular switch for X chromosome inactivation. Cell 143: 390-403.

101. Sripathy $S$ (2017) Screen for reactivation of MeCP2 on the inactive $X$ chromosome identifies the BMP/TGF-beta superfamily as a regulator of XIST expression. Proc Natl Acad Sci USA 114: 1619-1624.

102. Sirchia SM (2009) Misbehaviour of XIST RNA in breast cancer cells. PLoS One 4: e5559.

103.Zeng MN, Ma WL, Zheng WL (2016) Bioinformatics analysis of microRNA comprehensive regulatory network in B- cell acute lymphoblastic leukemia. Zhonghua Xue Ye Xue Za Zhi 37: 585-590.

104.Wang Z (2016) Long non-coding RNA TSIX is upregulated in scleroderma dermal fibroblasts and controls collagen mRNA stabilization. Exp Dermato 25: 131-136.

105. Chelmicki T (2014) MOF-associated complexes ensure stem cell identity and Xist repression. Elife 3: e02024.

106. Mlynarczyk SK, Panning B (2000) X inactivation: Tsix and Xist as yin and yang. Curr Biol 10: R899-903.

107. Shibata S, Yokota T, Wutz A(2008) Synergy of Eed and Tsix in the repression of Xist gene and X-chromosome inactivation. EMBO J 27: 1816-1826.

108. Kim J, Kim JD (2008) In vivo YY1 knockdown effects on genomic imprinting Hum Mol Genet 17: 391-401.

109. Pontier DB, Gribnau J (2011) Xist regulation and function explored. Hum Genet 130: 223-236.

110. Do JT (2008) Enhanced reprogramming of Xist by induced upregulation of Tsix and Dnmt3a. Stem Cells 26: 2821-2831.

111. Donohoe ME (2007) Identification of a Ctcf cofactor, Yy1, for the X chromosome binary switch. Mol Cell 25: 43-56.
112. Chao W (2002) CTCF, a candidate trans-acting factor for X-inactivation choice. Science 295: 345-347.

113. Morey C (2001) Tsix-mediated repression of Xist accumulation is not sufficien for normal random X inactivation. Hum Mol Genet 10: 1403-1411.

114. Brown CJ, Chow JC (2003) Beyond sense: The role of antisense RNA in controlling Xist expression. Semin Cell Dev Biol 14: 341-347.

115. Sado T, Hoki Y, Sasaki H (2005) Tsix silences Xist through modification of chromatin structure. Dev Cell 9: 159-165.

116. Migeon BR (2001) Identification of TSIX, encoding an RNA antisense to human XIST, reveals differences from its murine counterpart: Implications for X inactivation. Am J Hum Genet 69: 951-60.

117. Sun W (2017) Knockdown of IncRNA-XIST enhances the chemosensitivity of NSCLC cells via suppression of autophagy. Oncol Rep 38: 3347-3354.

118. Kobayashi R (2016) Increased expression of long non-coding RNA XIST predicts favorable prognosis of cervical squamous cell carcinoma subsequent to definitive chemoradiation therapy. Oncol Lett 12: 3066-3074.

119. Schouten PC (2016) High XIST and low 53BP1 expression predict poor outcome after high-dose alkylating chemotherapy in patients with a BRCA1 like breast cancer. Mol Cancer Ther 15: 190-198.

120. Huang KC (2002) Relationship of XIST expression and responses of ovarian cancer to chemotherapy. Mol Cancer Ther 1: 769-776.

121.Sun J (2017) LncRNA XIST promotes human lung adenocarcinoma cells to cisplatin resistance via let-7i/BAG-1 axis. Cell Cycle 16: 2100-2107.

122.Du P (2017) LncRNA-XIST interacts with miR-29c to modulate the chemoresistance of glioma cell to TMZ through DNA mismatch repair pathway. Biosci Rep 37.

123. Han Q (2017) Downregulation of IncRNA X inactive specific transcript (XIST) suppresses cell proliferation and enhances radiosensitivity by Upregulating mir-29c in nasopharyngeal carcinoma cells. Med Sci Monit 23: 4798-4807.

124.Song H (2017) Long non-coding RNA XIST functions as an oncogene in human colorectal cancer by targeting miR-132-3p. J BUON 22: 696-703.

125. Tang $Y(2017)$ IncRNA XIST interacts with miR-140 to modulate lung cance growth by targeting iASPP. Oncol Rep 38: 941-948.

126. Wang $H$ (2017) The long non-coding RNA XIST controls non-small cell lung cancer proliferation and invasion by modulating miR-186-5p. Cell Physiol Biochem 41: 2221-2229.

127.Fang J, Sun CC, Gong C (2016) Long non-coding RNA XIST acts as an oncogene in non-small cell lung cancer by epigenetically repressing KLF2 expression. Biochem Biophys Res Commun 478: 811-817.

128. Tantai $\mathrm{J}$ (2015) Combined identification of long non-coding RNA XIST and HIF1A-AS1 in serum as an effective screening for non-small cell lung cancer Int J Clin Exp Pathol 8: 7887-7895.

129. Lv GY, Miao J, Zhang XL (2017) Long non-coding RNA XIST promotes osteosarcoma progression by targeting Ras-related protein RAP2B via miR320b. Oncol Res 1: 1-2.

130. Chang S (2017) Long non-coding RNA XIST regulates PTEN expression by sponging miR-181a and promotes hepatocellular carcinoma progression. BMC Cancer 17: 248.

131.Zhuang LK (2016) MicroRNA-92b promotes hepatocellular carcinoma progression by targeting Smad7 and is mediated by long non-coding RNA XIST. Cell Death Dis 7: e2203

132. Christie MJ (1987) Excitatory amino acid projections to the nucleus accumbens septi in the rat: a retrograde transport study utilizing $\mathrm{D}[3 \mathrm{H}]$ aspartate and $[3 \mathrm{H}]$ GABA. Neuroscience 22: 425-439.

133. Wei W (2017) LncRNA XIST promotes pancreatic cancer proliferation through miR-133a/EGFR. J Cell Biochem 118: 3349-3358.

134.Tzeng CW (2007) EGFR genomic gain and aberrant pathway signaling in pancreatic cancer patients. J Surg Res 143: 20-26.

135. Yarden $Y$, Sliwkowski MX (2001) Untangling the ErbB signalling network. Nat Rev Mol Cell Biol 2: 127-137. 
Citation: Samir A, Salama E, El-Tayebi HM (2018) The Long Non-Coding RNA XIST: A New Cornerstone in Carcinogenesis. J Mol Genet Med 12: 356 doi:10.4172/1747-0862.1000356

136. Ma L (2017) Long non-coding RNA XIST promotes cell growth and invasion through regulating miR-497/MACC1 axis in gastric cancer. Oncotarget 8 : 4125-4135.

137. Chen DL (2016) Long non-coding RNA XIST regulates gastric cance progression by acting as a molecular sponge of miR-101 to modulate EZH2 expression. J Exp Clin Cancer Res 35: 142.

138.Zhong $Y$ (2016) Dysregulated expression of long noncoding RNAs in ovarian cancer. Int J Gynecol Cancer 26: 1564-1570.

139.Song P (2016) Long non-coding RNA XIST exerts oncogenic functions in human nasopharyngeal carcinoma by targeting miR-34a-5p. Gene 592: 8-14.

140.Salvador MA (2013) The histone deacetylase inhibitor abexinostat induces cancer stem cells differentiation in breast cancer with low Xist expression. Clin Cancer Res 19: 6520-6531.

141. Yao Y (2015) Knockdown of long non-coding RNA XIST exerts tumorsuppressive functions in human glioblastoma stem cells by up-regulating miR152. Cancer Lett 359: 75-86.

142. Kawakami T (2004) XIST unmethylated DNA fragments in male-derived plasma as a tumour marker for testicular cancer. Lancet 363: 40-42.

143. Laner T (2005) Hypomethylation of the XIST gene promoter in prostate cancer. Oncol Res 15: 257-264.

144. Sakamoto KM (2004) Knocking down human disease: Potential uses of RNA interference in research and gene therapy. Pediatric Research 55: 912-913.

145. Noll B, Seiffert S, Vornlocher HP (2011) Roehl I: Characterization of small interfering RNA by non-denaturing ion-pair reversed-phase liquid chromatography. J Chromatogr A 1218: 5609-5617

146. Martinez J, Patkaniowska A, Urlaub H, Lührmann R, Tuschl T (2002) Single stranded antisense siRNAs guide target RNA cleavage in RNAi. Cell 110: 563-574.

147. Martinez J, Tuschl T (2004) RISC is a 5' phosphomonoester-producing RNA endonuclease. Genes Dev 18: 975-980.

148. Vassilev LT (2004) Small-molecule antagonists of p53-MDM2 binding: research tools and potential therapeutics. Cell Cycle 3: 419-421.

149. Brennecke J, Aravin AA, Stark A, Dus M, Kellis M, et al. (2007) Discrete small RNA-generating loci as master regulators of transposon activity in Drosophila. Cell 128: 1089-1103.

150. Bortolin ML, Kiss T (1998) Human U19 intron-encoded snoRNA is processed from a long primary transcript that possesses little potential for protein coding. RNA 4: 445-454.

151. Ganot P, Caizergues-Ferrer M, Kiss T (1997) The family of box ACA small nucleolar RNAs is defined by an evolutionarily conserved secondary structure and ubiquitous sequence elements essential for RNA accumulation. Genes Dev 11: 941-956

152. Kiss T (2002) Small nucleolar RNAs: An abundant group of noncoding RNAs with diverse cellular functions. Cell 109: 145-148.

153. Ender C, Krek A, Friedländer MR, Beitzinger M, Weinmann L, et al. (2008) A human snoRNA with microRNA-like functions. Mol Cell 32: 519-528.

154. Chang LS, Lin SY, Lieu AS, Wu TL (2002) Differential expression of human $5 \mathrm{~S}$ snoRNA genes. Biochemical and Biophysical Research Communications 299: 196-200.

155. Hawkins PG, Santoso S, Adams C, Anest V, Morris KV (2009) Promoter targeted small RNAs induce long-term transcriptional gene silencing in human cells. Nucleic Acids Res 37: 2984-2995.
156. Carone DM, Longo MS, Ferreri GC, Hall L, Harris M, et al. (2009) A new class of retroviral and satellite encoded small RNAs emanates from mammalian centromeres. Chromosoma 118: 113-125.

157. Meynert A, Birney E (2006) Picking pyknons out of the human genome. Cell 125: 836-838.

158. Rigoutsos I, Huynh T, Miranda K, Tsirigos A, McHardy A, et al. (2006) Short blocks from the noncoding parts of the human genome have instances within nearly all known genes and relate to biological processes. Proc Natl Acad Sci USA 103: 6605-6610.

159. Stabenau A, McVicker G, Melsopp C, Proctor G, Clamp M, et al. (2004) The ensemble core software libraries. Genome Res 14: 929-933.

160. Fei Liu, Chen NA, Yanchun G, Ruihai X, Weichao W, et al. (2017) The long non-coding RNA NEAT1 enhances epithelial-to-mesenchymal transition and chemoresistance via the miR-34a/ c-Met axis in renal cell carcinoma Oncotarget 1: 1-12

161. Wu X, Dinglin X, Wang X, Luo W, Shen Q, et al. (2017) Long noncoding RNA XIST promotes malignancies of esophageal squamous cell carcinoma via regulation of $\mathrm{miR}-101 / \mathrm{EZH} 2$. Oncotarget 1: 1-2.

162. Shun WC, Jin LH, Jing C, Yan WH, Xiu MH, et al. (2017) Long non-coding RNA UBE2CP3 promotes tumor metastasis by inducing epithelial-mesenchymal transition in hepatocellular carcinoma, Oncotarget 1: 1-16.

163. Gabet Y, Baniwal SK, Leclerc N, Shi Y, Gabe KAE, et al. (2010) Krox20/EGR2 deficiency accelerates cell growth and differentiation in the monocytic lineage and decreases bone mass. Blood 116: 3964-3971.

164. Xiao J, Ding Y, Huang J, Li Q, Liu Y, et al. (2001) The association of HMGB1 gene with the prognosis of HCC. PLoS One 9: e89097.

165. Chen RC, Yi PP, Zhou RR, Xiao MF, Huang ZB, et al. (2014) The role of HMGB1-RAGE axis in migration and invasion of hepatocellular carcinoma cell lines. Mol Cell Biochem 390: 271-280.

166. Cordenonsi M, Zanconato F, Azzolin L, Forcato M, Rosato A, et al. (2011) The Hippo transducer TAZ confers cancer stem cell-related traits on breast cancer cells. Cell 147: 759-772.

167. Raman V, Martensen SA, Reisman D, Evron E, Odenwald WF, et al. (2000) Compromised HOXA5 function can limit p53 expression in human breast cancer. Nature 405: 974-978.

168. Chenhui Ma, Wu G (2017) Long intergenic noncoding RNA 00673 promotes non-small-cell lung cancer metastasis by binding with EZH2 and causing epigenetic silencing of HOXA5. Oncotarget 8: 32696-32705.

169. Leeman MF, Curran S, Murray GI (2002) The structure, regulation, and function of human matrix metalloproteinase-13. Crit Rev Biochem Mol Biol 37: 149-166.

170. Kan H, Guo W, Huang Y, Liu D (2015) MicroRNA-520 g induces epithelialmesenchymal transition and promotes metastasis of hepatocellular carcinoma by targeting SMAD7. FEBS Lett 589: 102-109.

171. Mora A, Komander D, Van Aalten DM (2004) PDK1, the master regulator of AGC kinase signal transduction. Semin Cell Develop Biol 15: 161-170.

172. Gao Y, Wu F, Zhou J (2014) The H19/let-7 double-negative feedback loop contributes to glucose metabolism in muscle cells. Nucleic Acid Res 42 13799-13811.

173. Nakamura K, Sakaue H, Nishizawa A (2008) PDK1 regulates cell proliferation and cell cycle progression through control of cyclin D1 and p27Kip1 expression. J Biol Chem 283: 17702-17711. 